\title{
Transneuronal Dpr12/DIP- $\delta$ interactions facilitate compartmentalized dopaminergic innervation of Drosophila mushroom body axons
}

Bavat Bornstein ${ }^{1}$, Idan Alyagor ${ }^{1}$, Victoria Berkun ${ }^{1}$, Hagar Meltzer ${ }^{1}$, Fabienne Reh², Hadas Keren-Shaul ${ }^{3,4}$, Eyal David ${ }^{3}$, Thomas Riemensperger ${ }^{2}$ and Oren Schuldiner ${ }^{{ }^{*}}$

${ }^{1}$ Department of Molecular Cell Biology, Weizmann Institute of Sciences, Rehovot, 15 Israel

2 Institute of Zoology, University of Cologne, Köln, Germany

${ }^{3}$ Department of Immunology, Weizmann Institute of Sciences, Rehovot, Israel

${ }^{4}$ Life Science Core Facility, Weizmann Institute of Sciences, Rehovot, Israel

* Corresponding Author and Lead Contact:

e-mail oren.schuldiner@weizmann.ac.il

Keywords: Neuronal remodeling; Mushroom body; compartmentalized innervation; mushroom body compartments; dopaminergic neurons; Dpr; DIP; IgSF; Drosophila 


\section{Summary}

The mechanisms controlling wiring of neuronal networks are largely unknown. The stereotypic architecture of the Drosophila mushroom-body (MB) offers a unique system to study circuit assembly. The adult medial MB $y$-lobe is comprised of a long bundle of axons that wires with specific modulatory and output neurons in a tiled

30 manner defining five distinct zones. We found that the immunoglobulin superfamily protein Dpr12 is cell-autonomously required in y-neurons for their developmental regrowth into the distal $\mathrm{Y} 4 / 5$ zones, where both Dpr12 and its interacting protein, DIP- $\delta$, are enriched. DIP- $\delta$ functions in a subset of dopaminergic neurons that wire with $\mathrm{y}$-neurons within the $\mathrm{\gamma} 4 / 5$ zone. During metamorphosis, these dopaminergic projections arrive to the $\gamma 4 / 5$ zone prior to $\gamma$-axons, suggesting that $\gamma$-axons extend through a prepatterned region. Thus, Dpr12/DIP- $\delta$ transneuronal interaction is required for $\mathrm{y} 4 / 5$ zone formation. Our study sheds light onto molecular and cellular mechanisms underlying circuit formation within subcellular resolution. 


\section{Introduction}

The precise connectivity between neurons is crucial for the function of neural circuits in vertebrates and invertebrates. The formation of neural circuits is especially complex as it is a multi-step process that involves guidance of axons and dendrites belonging to distinct neurons, as well as the identification of subcellular zones on the target cell onto which synapses are formed. Despite its fundamental nature, the molecular and cellular mechanisms underlying development of neural circuits remain mostly poorly understood.

Given its unique development, connectivity and function, the Drosophila mushroom body (MB), offers an attractive model to study the mechanisms of neuronal circuit formation and maturation. The adult MB, implicated in associated learning (Fiala, 2007; Gerber et al., 2004; Heisenberg, 2003; Modi et al., 2020; Owald and Waddell, 2015), is comprised of intrinsic as well as extrinsic neurons (Aso et al., 2014a; Tanaka et al., 2008). Intrinsic MB neurons are derived from four identical neuroblasts which sequentially give rise to three major classes of unipolar neurons: $\gamma, \alpha^{\prime} / \beta^{\prime}$ and $\alpha / \beta$, which are collectively known as Kenyon Cells (KCs). Axons from each KC type bundle together to form five MB lobes in the adult brain - the vertical $\alpha$ and $\alpha$ ' lobes and the medial $\gamma, \beta$ and $\beta$ ' lobes (Figure 1A, Crittenden et al., 1998). KCs form well-defined circuits with MB extrinsic neurons, which include MB output neurons (MBONs) and modulatory neurons, mostly dopaminergic (DANs). The processes of MBONs and

60 DANs innervate the MB lobes at distinct and stereotypic locations thereby forming discrete zones, which are also known as compartments (Due to a potential confusion between cell intrinsic compartments such as the axon initial segment, here we use the term zone to describe these lobe-compartments; Aso et al., 2014a; Tanaka et al., 2008). For example, the $\mathrm{Y}$-lobe, which is comprised of $\mathrm{Y}-\mathrm{KC}$ axons, is innervated by extrinsic neurons in five distinct axonal zones termed $\mathrm{y} 1-\mathrm{y} 5$. Thus, each $y$-zone is defined by stereotypic inputs from specific DANs and MBONs (Figure 1A). Each $Y-$ axon extends throughout the entire lobe and forms synaptic boutons with different partners within each zone. Remarkably, a recent study has shown that boutons within the same KC, but in different zones, often exhibit distinct calcium dynamics (Bilz et al., 2020). Finally, these zones have distinct functional roles. DANs innervating the $\mathrm{y} 1-\mathrm{\gamma} 2$ zones are associated with aversive memory, while DANs innervating the $\mathrm{y} 4-5$ zones promote appetitive memory (Aso et al., 2014a; Cognigni et al., 2018; Cohn et al., 
2015). Despite the functional importance, the cellular and molecular mechanisms that control MB circuit and zone formation are not known.

75 The MB is attractive to study wiring of neural circuits not only due to its complex yet stereotypic nature, but also due to its multi-step development. The larval MB is primarily comprised of $\mathrm{Y}$-KCs which form two axonal lobes (a vertical and medial $\mathrm{Y}$ lobes). These are innervated by MBONs and DANs in distinct zones that are different from the adult pattern of zonation (Rohwedder et al., 2016; Saumweber et

80 al., 2018). Subsequently, the y-lobes undergo extensive remodeling during metamorphosis, including axon pruning followed by developmental regrowth, (Lee et al., 1999) (Figure 1B) to give rise to the adult medial Y-lobe containing the Y1-5 zones (Figure 1A). We have previously demonstrated that regrowth of the adult $\mathrm{Y}$ lobe is genetically controlled by the nuclear receptor Unfulfilled (UNF) functioning as a ligand dependent transcription factor by mechanisms distinct from initial axon outgrowth (Yaniv et al., 2012). Importantly, while we found that UNF promotes axon regrowth partly via the TOR pathway, it is yet unclear through which mechanisms it promotes targeting, circuitry and sub-zone formation. Here, we exploit detailed expression profile analyses to focus on the Immunoglobulin superfamily (IgSF) proteins as potential mediators of zone formation and circuit wiring within the $\mathrm{\gamma} 4 / 5$ zones.

\section{Results}

\section{Dpr12 is required for $y$ neuron regrowth}

95 To identify potential genes and pathways that mediate axon regrowth and circuit formation, we sequenced the RNA content of WT $\mathrm{Y}-\mathrm{KC}$ s during development (Alyagor et al., 2018) alongside $\mathrm{Y}$-KCs expressing RNAi targeting UNF, a known protein required for regrowth (Figure 1B). Supplementary Table 1 shows this comparison alongside the previously generated (Alyagor et al., 2018) expression

100 profiles of $\mathrm{y}$ neurons expressing a dominant negative form of the Ecdysone Receptor $\left(E c R^{D N}\right)$, which is required for pruning (Lee et al., 2000; data is freely available in: https://www.weizmann.ac.il/mcb/Schuldiner/resources). The immunoglobulin superfamily (IgSF) appeared as the protein family most significantly affected by UNF RNAi expression ( $p=3^{*} 10^{-29}$; analyzed in http://www.flymine.org/). Within the lgSF, 
105 the Defective Proboscis Response (Dpr) family stood out as 16 of 21 members were significantly expressed in dynamic patterns in developing Y-KCs (Figure 1C, Supplementary Table 2). We found that the transcription of approximately half of the Dprs (7/16; supplemental Table 2) was significantly reduced in UNF-RNAi expressing flies. Interestingly, the interactions between the Dprs, containing two

110 immunoglobulin (lg) domains, and the Dpr Interacting Proteins (DIPs), containing three Ig domains, are important for proper development and synaptic connectivity of the Drosophila visual system and neuromuscular junction (NMJ, Ashley et al., 2019; Venkatasubramanian et al., 2019; Xu et al., 2018). Based on these data, we focused on the Dprs as potential candidates required for regrowth and circuit formation.

115 We therefore targeted 8 different Dprs in $\mathrm{Y}$-KCs by RNAi (Figure S1A) based on reagent availability (TRiP lines, https://fgr.hms.harvard.edu/fly-in-vivo-rnai). Seven of these RNAi's did not affect $y$ neuron development. At this time we cannot conclude whether these Dprs are indeed not required for $\mathrm{Y}$-KCs development or, alternatively, that the lack of phenotype is due to inherent redundancies of Dpr-DIP interactions

120 (Cosmanescu et al., 2018). In contrast, expressing dpr12 RNAi in Y-KCs induced a dramatic regrowth defect, where axons did not occupy the distal portion of the lobe (Figures 1D-E, S1A). Interestingly, the expression of Dpr12 is dramatically reduced in neurons expressing UNF-RNAi at the relevant times for regrowth (Figures 1C, S1A, and Supplementary Table 2), suggesting that UNF might positively regulate $125 d p r 12$ transcription. These findings suggest that Dpr12 could promote developmental regrowth and circuit formation as a part of an UNF dependent transcriptional program.

To validate the RNAi results, we next perturbed Dpr12 through tissue specific (ts)CRISPR using Gal4 driven Cas9 expression (Meltzer et al., 2019; Port and

130 Bullock, 2016; Port et al., 2020). tsCRISPR of dpr12 in Y-KCs induced a defect closely resembling the RNAi phenotype (Figure 1F-G). Finally, we used CRISPR/Cas9 technology to generate a dpr12 loss of function mutant (dpr12 ${ }^{\Delta 50-81}$, Figure S1B-C). At $3^{\text {rd }}$ instar larva (L3), $\mathrm{Y}-\mathrm{KC}$ in $d p r 12^{\Delta 50-81}$ homozygotes exhibited WT morphology (Figure 1H, L) and subsequently pruned normally (data not shown).

135 These data indicate that Dpr12 is not required for initial axon extension or pruning. In contrast, $\mathrm{Y}$-KCs in $d p r 12^{\Delta 50-81}$ animals failed to extend to the distal part of the lobe at 48h APF, a time when $\mathrm{y}$-axons would have normally completed their regrowth (Lee 
et al., 1999; Rabinovich et al., 2016), and in adult (Figure 1I,J, M, N). Importantly, Yspecific expression of a Dpr12 transgene significantly rescued the regrowth defect

140 within $d p r 12^{\Delta 50-81}$ homozygotes (Figure $1 \mathrm{~K}, \mathrm{O}, \mathrm{P}$, ranking examples shown in Figure S1D). These data demonstrate that Dpr12 is required for axon regrowth during metamorphosis and that mutant $\mathrm{y}$-axons stop prematurely and do not extend into the distal end of the lobe.

145 Dpr12 is cell autonomously required for $\mathrm{y}$-axon regrowth into the $\mathrm{y} 4 / 5$ zones

To determine whether Dpr12 functions in a cell-autonomous manner, we used the Mosaic Analysis with a Repressible Cell Marker (MARCM) technique to express dpr12-RNAi within neuroblast (NB) or single cell (SC) clones. We found that both NB and single $\mathrm{Y}-\mathrm{KC}$ clones expressing dpr12 RNAi exhibited normal growth at L3 but

150 failed to fully extend axons at 48hr APF and in adult flies (Figure 2A-L). Based on these results, we conclude that Dpr12 is cell-autonomously required in $\mathrm{y}-\mathrm{KCs}$ for their full developmental regrowth.

Interestingly, unlike other mutants that affect developmental regrowth (Yaniv et al., 2012; Yaniv et al., 2020) dpr12 mutant axons seem to partially regrow but stop prematurely in a particular and stereotypic location along the lobe. Therefore, and given that the $\mathrm{Y}$-lobe is divided into distinct zones, we next mapped the location of the premature stopping in more detail. We measured the length of adult WT and mutant axons relative to the $y$-lobe span and superimposed these data onto the $y$ lobe zones (Figures 2M, S2), as defined by distinct innervations of MBONs and

160 DANs (Aso et al., 2014a; Shuai et al., 2015). This analysis indicated that the premature stopping of clones expressing dpr12 RNAi correlates with the border between the $\mathrm{Y} 3$ and $\mathrm{y} 4$ zones (Figures 2l', L', N, Supplemental movies 1-2). Interestingly, we found that approximately $70 \%$ of SC clones stopped at the $\mathrm{\gamma} 3 / 4$ border, while the remaining clones extended to the end of the lobe (Figure $2 \mathrm{~N}$ ).

165 Since axon stalled at a discrete location, our data suggest that the phenotype does not arise from reduced growth potential, per se, but rather through a failure to recognize a molecular signal at a designated and stereotypic location. We conclude that Dpr12 is cell-autonomously required for $\mathrm{Y}-\mathrm{KC}$ projection into the $\mathrm{MB} \mathrm{\gamma} 4 / 5$ zones. 


\section{Dpr12 and its putative interacting protein DIP- $\delta$ localize at the $y 4 / 5$ zones}

To assess Dpr12 protein localization, we used the Minos Mediated Integration Cassette transgene collection to generate a GFP insertion within the endogenous Dpr12 locus, which should produce a Dpr12-GFP fusion protein (Dpr12GFSTF;

Figure S1B-C; Nagarkar-Jaiswal et al., 2015). We found that Dpr12-GFP localized to 175 the distal part of $\mathrm{y}$-axons at late larva (L3; Figure 3A), then becoming diffuse at $24 \mathrm{hr}$ APF, when $\mathrm{y}$-axons initiate their developmental regrowth (Figure 3B). Finally, at 48h APF and in adulthood, Dpr12 relocalized to the distal part of the lobe (Figure 3C-D) and was restricted to the adult $\mathrm{\gamma} 4$ and $\mathrm{\gamma} 5$ zones (Figure 3D). Our data suggest that Dpr12 is expressed at the right time and place to mediate $\mathrm{Y}$-axon regrowth into the ү4/y5 zones.

Dprs can form heterophilic interactions with DIPs in a rather promiscuous fashion, in which most Dprs can bind to multiple DIPs and most DIPs can bind to multiple Dprs (Carrillo et al., 2015; Cosmanescu et al., 2018; Ozkan et al., 2013). Interestingly, Dpr12 and DIP- $\delta$ represent a unique case of 'monogamous' binding. We found that DIP- $\delta-G F P$ (DIP- $\delta$ GFSTF; Figure S3A-B) localized, similarly to Dpr12, to the $\gamma 4 / 5$ zones (Figure $3 \mathrm{H}$ ). However, in contrast to Dpr12, DIP- $\delta$ was localized to the distal MB lobe at all developmental timepoints tested (Figure 3E-H), including at 24hr APF, when $\mathrm{Y}$-axons are completely pruned and have not yet extended (Figure 3F). Taken together, these data indicate that DIP- $\delta$ is localized to $\gamma 4 / \gamma 5$ zones throughout

190 development and is expressed in cells that project to this region before $\mathrm{Y}$-KCs reach their terminal projections.

\section{DIP- $\delta$ is non-cell autonomously required for $y$-axon regrowth}

We next asked whether DIP- $\delta$ is also required for the extension of $\gamma$-axons into the Y $4 / 5$ zones. We therefore both generated and obtained DIP- $\delta$ mutant alleles (DIP$\delta^{T 2 A-G a l 4}, D I P-\delta^{1-119}$, respectively; Figure S3A-B). We marked the $\mathrm{Y}$-KCs by expressing a membrane bound Tomato (QUAS-mtdT-3XHA) driven by the Gal4independent $\gamma-\mathrm{KC}$ specific QF2 driver (71G10-QF2). At L3, Y-KCs within DIP- $\delta$ mutant brains exhibited WT morphology (Figure 4A, E, S3C), indicating that DIP- $\delta$ is not required for their initial axon extension. However, DIP- $\delta$ mutant brains displayed a $\mathrm{y}$-axon regrowth defect at 48hr APF, and in adult (Figure 4B, C, F, G, Figure S3D) 
which resembled the dpr12 mutant phenotype. To confirm that DIP- $\delta$ loss-of-function induced this $\mathrm{Y}$-axon regrowth defect, we exploited the fact that the DIP- $\delta^{T 2 A-G a l 4}$ allele also expresses Gal4 in DIP- $\delta^{+}$neurons (Figure S3A-B). Expressing DIP- $\delta$ transgene driven by DIP- $\delta^{T 2 A-G a l 4}$ rescued the $y$-axon extension defect (Figure 4D, H, I, S3E) confirming that DIP- $\delta$ is required for $\mathrm{Y}$-axon innervation of the $\gamma 4 / 5$ zones.

To identify these DIP-ס expressing cells, we expressed DIP- $\delta$ RNAi in different cell types, while simultaneously labeling Y-KCs using the QF2 system described above. Driving the expression of DIP- $\delta$-RNAi in all glia (using the Pan-glial driver Repo-

210 Gal4) or all KCs (using OK107-Gal4) did not affect y-axon extension (Figure S4A-B). In contrast, knocking down of DIP- $\delta$ in all neurons (using the pan-neuronal driver C155-Gal4) or all DIP- $\delta$ expressing neurons (using DIP- $\delta^{T 2 A-G a / 4}$ ) resulted in stalled $\mathrm{Y}$-axons that do not innervate the $\mathrm{Y} 4 / 5$ zones (Figure S4C-D). Similarly, tsCRISPR of $D I P-\delta$ in all neurons, but not when restricted to $y$ neurons, affected the extension of

$215 Y$-axons (Figure S4E-H). Together, these experiments indicate that DIP- $\delta$ is not required in $y$ or other KCs. Rather, DIP- $\delta$ likely functions in extrinsic MB neurons in a non-cell autonomous manner to mediate the $y$-axon extension into the $\mathrm{y} 4 / 5$ zones.

\section{A subpopulation of DANs express DIP- $\delta$ in the $y 4 / y 5$ zones}

220 Our data suggest that DIP- $\delta$ is expressed in extrinsic MB neurons that innervate the Y4/Y5 zones (Figures 3,4). These zones are strongly innervated by the Protocerebral Anterior Medial (PAM) neurons, a population of about 100 DANs, as well as by specific groups of MBONs (Aso et al., 2014a; Tanaka et al., 2008). To investigate whether DIP- $\delta$ is expressed in these cells, we selectively ablated PAM-DANs or

225 MBONs by cell type specific expression of Diphtheria toxin (UAS-DTI) and assayed DIP-ס-GFP localization in adult flies. Ablating one of the $\mathrm{Y} 4 \mathrm{MBONs}$ (MBONY4> Y1Y2) did not affect DIP- $\delta$ expression (Figure 5A-B). In contrast, ablating PAM DANs using R58E02-Gal4 drastically reduced $ү 4 / 5$ specific DIP- $\delta$ expression (Figure $5 C-D)$. While we cannot exclude the possibility that DIP- $\delta$ is additionally expressed in 230 the other $\mathrm{Y} 4 \mathrm{MBON}(\mathrm{MBON}-\mathrm{\gamma} 4 \mathrm{\gamma 5})$, these results strongly suggest that at the adult stage, DIP- $\delta$ protein that is localized to the $\gamma 4 / 5$ compartments is mainly, if not exclusively, expressed by PAM DANs, consistent with recent profiling experiments (Croset et al., 2018). 
Next, we visualized PAM-DANs during development to determine if they may provide a template for $\gamma$-axon growth, as suggested by DIP-ס-GFP expression (Figure 3).

Since the 'classical' PAM driver, R58E02, is not expressed throughout development (Figure S5A-C, and data not shown), we analyzed the expression of DIP- $\delta^{T 2 A-G a l 4}$ (Figure S5D-F) and then used the MARCM technique to label sparse DIP- $\delta^{T 2 A-G a / 4}$ clones (Figure 5E-G; of note, these clones remain heterozygous for DIP-ס). We

240 detected DIP- $\delta$ expressing clones that innervate the $\mathrm{\gamma} 4 / \mathrm{\gamma} 5$ zones as early as $24 \mathrm{hr}$ APF and up to adulthood. Interestingly, these clones do not seem to express tyrosine hydroxylase (TH), required for Dopamine biogenesis, at $24 \mathrm{hr}$ APF but become TH positive at 48hr APF onwards. In summary, our data suggest that DIP- $\delta$ is expressed in PAM-DANs, which innervate the future $\mathrm{Y} 4 / 5$ zones as early as $24 \mathrm{hr}$

245 APF, and support the speculation that DIP- $\delta$ expressed in PAM-DANs may provide a template for $\mathrm{y}$-axon growth.

\section{DIP-ס is required and sufficient for Dpr12 localization}

Both Dpr12 and DIP- $\delta$ localize to the $\mathrm{Y} 4 / 5$ zones and are required for their formation likely by mediating interactions between the $\mathrm{Y}-\mathrm{KC}$ s and the PAM-DANs. We therefore investigated how losing either $d p r 12$ or DIP- $\delta$ affects their binding partner localization and mature circuit architecture. First, we investigated whether the highly localized expression of Dpr12 and DIP- $\delta$ is cell-autonomous or requires interaction with their binding partner. We visualized Dpr12- and DIP-ס-GFP fusion proteins in

255 brains homozygous mutant for their reciprocal Dpr/DIP partner. We found that Dpr12 expression appears diffuse in DIP- $\delta$ mutant brains throughout development (Figure 6A-D), indicating that Dpr12 protein localization requires interaction with DIP- $\delta$, likely on PAM-DANs. In contrast, DIP-ס localization did not dramatically change during the early stages of development in dpr12 mutants (Figure 6E-F). At 48hr APF, while we

260 still detected DIP- $\delta$ at the distal part of the lobe, it occupied a smaller area than in WT brains and resembled the innervation pattern typical of $24 \mathrm{hr}$ APF (compare Figure $6 G$ to Figure 3G). Furthermore, we did not detect any DIP- $\delta$ in the adult MB medial lobe (Figure $6 \mathrm{H}$ ), suggesting that Dpr12 is required for the refinement and maintenance of DIP-ס localization. 
localization, we mis-expressed DIP- $\delta$ in DANs that innervate $\gamma 3$ zone, normally devoid of Dpr12 protein (Figure 6I). Remarkably, we found that DIP-ס misexpression indeed caused Dpr12 protein to become localized to the adult $\mathrm{\gamma} 3$ zone, in addition to its endogenous $\mathrm{\gamma} 4 / \mathrm{\gamma} 5$ expression (Figure 6J). Together, these results indicate that

270 while DIP- $\delta$ is both required and sufficient for Dpr12 localization throughout development, Dpr12 is required only for maintenance of the adult localization of DIP$\delta$.

\section{Dpr12-DIP-ס interaction mediates circuit re-assembly}

We next determined if the loss of normal Dpr12-DIP-ס interaction induced axonal misrouting, cell loss or other circuit reorganizations. In dpr12 mutants, we found that PAM-DANs which normally target the $\mathrm{y} 4$ or $\mathrm{\gamma} 5$ zones misrouted and failed to form substantial connections within the Y-lobe (Figure 7A-D, Figure S6). Importantly, the number of PAM-DAN cell bodies of the subtypes tested remained unchanged (Figure

280 S6C, F). In contrast, Y4-MBON still innervated the Y-lobe in dpr12 mutant brains, albeit in abnormal locations like the $\mathrm{Y} 3$ compartment (Figure 7E-F, Figure S6). Finally, we examined the global neuropil structure in $d p r 12$ mutant brains by following staining of the active zone protein bruchpilot (Brp), which demonstrated that the $\mathrm{y} 4 / 5$ zones were largely missing (Figure 7G-H, Movies S3-4). Interestingly, the lack of these domains was accompanied by enlarged $\mathrm{y} 2 / 3$ zones, as well as distortions in other brain regions (see Crepine, for example, in Figure 7G-H).

Taken together, our results suggest that the Dpr12-DIP- $\delta$ interaction is required for $\mathrm{Y} 4 / 5$ zone formation by mediating interactions between $\mathrm{Y}-\mathrm{KCs}$ and a subpopulation of PAM-DANs, eventually instructing their adult-specific connectivity.

\section{Discussion}

Our understanding of the development of complex neural circuits remains largely unknown. Specifically, how long axons can make en passant synapses with different partners in a stereotypic manner is not well understood. The unique development and morphology of the Drosophila MB y-lobe, combined with the awesome genetic power of the fly, offers an excellent opportunity to dissect mechanisms required for wiring of complex neural network and specifically for mechanisms that drive zonation 
within axonal bundles to allow for stereotypic localized innervation by distinct populations of neurons. Here, we identify a molecular mechanism that mediates neuron-neuron interactions that subsequently promote the formation of stereotypic circuits that define subcellular axonal zones.

The adult $\mathrm{y}$-lobe is divided into zones (also known as compartments) due to specific and localized innervations by extrinsic MB neurons including MBON and DANs. Here we show that the interaction between two IgSF proteins, Dpr12 on $\mathrm{y}-\mathrm{KCs}$ and DIP- $\delta$

305 on PAM-DANs, underlies the formation of MB $\mathrm{y} 4 / 5$ zones. Within each zone, input from DANs can modify synaptic strength between the $\mathrm{KC}$ and MBON to provide specific valence to sensory information(Aso et al., 2014a; Aso et al., 2014b; Cognigni et al., 2018; Cohn et al., 2015). Based on the results presented here, we speculate that various specific combinations of adhesion molecules may mediate target

310 recognition events that occur between predefined pre- and post-synaptic pairs in other zones as well. $\mathrm{Y}$-neurons express a broad spectrum of IgSFs in tight temporal regulation (Supplementary Table 1; Alyagor et al., 2018), highlighting their potential role in circuit formation. However, many adhesion molecules, including Dpr/DIPs, can form promiscuous interactions, making their analyses challenging. Future

315 studies could use CRISPR/Cas9 technology to generate multi-gene mutations to further explore the adhesion code required for zone/compartment formation.

Here we used the interaction between Dpr12 and DIP- $\delta$ to study the development of the $\mathrm{\gamma} 4 / 5$ zones. Our developmental analyses have concluded that DIP-ס expressing PAM-DANs arrive to the region of the $\mathrm{y} 4 / 5$ zones long before $\mathrm{y}$-axons. Interestingly,

320 in dpr12 mutant animals PAM-DANs arrive to the right place, linger there for a while ( 48h APF) and then eliminate their $\mathrm{Y} 4 / 5$ innervations, while maintaining and even strengthening/broadening other connections in the MB vicinity. Therefore, it is attractive to speculate that $\mathrm{y}$-axons extend into a prepatterned lobe. More studies comparing the development of other compartment-specific DANs as well as MBONs 325 are however required.

Here we demonstrate that Dpr12 is cell-autonomously required in Y-KCs while DIP-ס is required on PAM-DANs for the formation of the $\mathrm{Y} 4 / 5$ zones. To the best of our knowledge, this is the first case in which a Dpr molecule was shown to be cellautonomously required for correct wiring. However, many unresolved questions remain: 1) Why do the $\mathrm{y}$-axons stop prematurely? One possibility is that axon growth 
into the $\mathrm{Y} 4 / 5$ zones depends on Dpr12-DIP- $\delta$ interaction either because they overcome a yet undiscovered inhibitory signal or because they are positively required for the progression of the growth cone. Alternatively, Dpr12-DIP-ס interaction could be important for the stabilization of the connections between $Y$ axons and DAN processes to result in the formation of the $\mathrm{y} 4 / 5$ zones. At $48 \mathrm{~h}$ APF, the large majority of $d p r 12$ mutant $\mathrm{y}$-axons do not innervate the $\mathrm{\gamma} 4 / 5$ zones, arguing against the stability hypothesis; 2) What are the signaling pathways that mediate Dpr/DIP targeting recognition? None of the Dprs or DIPs contain a large intracellular domain that is capable of signaling. Identifying the co-receptor/s is a critical step in

340 gaining a mechanistic understanding of axon targeting whether in the visual, motor or MB circuits. The Dpr12/DIP- $\delta$ interaction which we have uncovered to be required for the formation of specific MB zones is an attractive model to further delve into these mechanisms due to its robust phenotype; 3 ) What is the significance of the GPI anchor? Many of the Dprs and DIPs, including DIP- $\delta$ (data not shown) are predicted GPI anchored proteins, suggesting that they can be cleaved to create a secreted soluble form. Whether this is an important step in targeting has not yet been investigated. Interestingly, the vertebrate homologs of the DIPs, the IgLON subfamily (Zinn and Ozkan, 2017), are GPI anchored proteins that were shown to be cut by metalloproteinases to promote axonal outgrowth (Sanz et al., 2015 ).

350 Expression patterns of Dpr and DIP molecules in the neuromuscular junction (NMJ) (Carrillo et al., 2015) and visual system (Carrillo et al., 2015; Tan et al., 2015) suggested a model where these molecules instruct target cell specificity. Recent loss-of-function experiments strengthened this target specificity hypothesis, as the DIP- $\alpha$-Dpr10 interaction was shown to be important for motoneuron innervation of specific larval (Ashley et al., 2019) and adult (Venkatasubramanian et al., 2019) muscles, and DIP-a-Dpr10/Dpr6 interaction for specific layer targeting in the visual system (Xu et al., 2018). Our results suggest that mechanisms used to target axons and dendrites to specific cell types or layers may be further implicated to orchestrate the wiring of long axons to different pre- and post-synaptic partners along their route and thus the formation of axonal zones.

Here we describe that interaction between two IgSF proteins mediates transneuronal communication that is required for proper wiring within specific zones of the Drosophila MB. The anatomical organization of the MB suggests that these 
interactions may provide target specificity for the long $\mathrm{KC}$ axon while it forms en passant synapses with different targets along its length. While the existence of such wiring architecture is known from invertebrates such as Drosophila and C. elegans, long axons making distinct yet stereotypic en passant connections is not widely described in vertebrates. Given the existence of long axons, that travel through dense neuropil structures, such as mossy fibers in the hippocampus, cholinergic

370 axons in the basal forebrain, and parallel fibers in the cerebellar cortex, we posit that this type of connectivity exists in vertebrates but has not yet been described in detail due to technological limitations that are likely to be resolved soon. Pairwise IgSF molecular interactions are conserved in vertebrates and invertebrates, implying similar mechanisms to dictate axon and dendrite targeting of subcellular neurite

375 zones in other organisms.

\section{Acknowledgments:}

We thank Larry Zipursky for sharing unpublished reagents, the Bloomington Stock Centers for reagents; monoclonal antibodies were obtained from the Developmental

380 Studies Hybridoma Bank developed under the auspices of the NICHD and maintained by the University of lowa. We thank R. Rothkopf for assistance with statistics; M. Schuldiner, A. Yaron, T. Misgeld and the O.S lab for discussions and critical reading of this manuscript. We thank Life Science Editors for editing assistance. Funding: This work was supported by the European Research Council

385 (erc), consolidator grant \# 615906, “AxonGrowth” and the Volkswagen Stiftung (joint Lower Saxony - Israel) grant \# ZN3459. Fly food for this project was funded by the Women Health Research Center. O.S. is the Incumbent of the Prof. Erwin Netter Professorial Chair of Cell Biology, T.R. was supported by Axa as team member of the Axa Chair from genome to structure, F.R is a GSfBS member and was supported

390 by Evangelisches Studienwerk Villigst e.V. Competing interests: Authors declare no competing interests. Data and materials availability: All data is available in the main text or the supplementary materials. In addition, RNAseq data will be available via Gene Expression Omnibus at NCBI. 
Bornstein et al. Figure 1

A

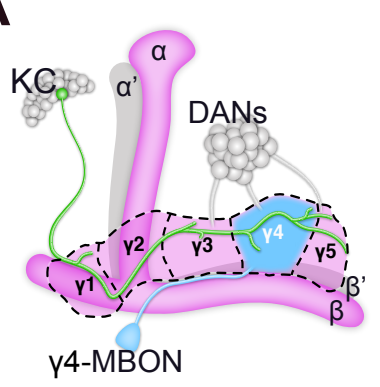

B
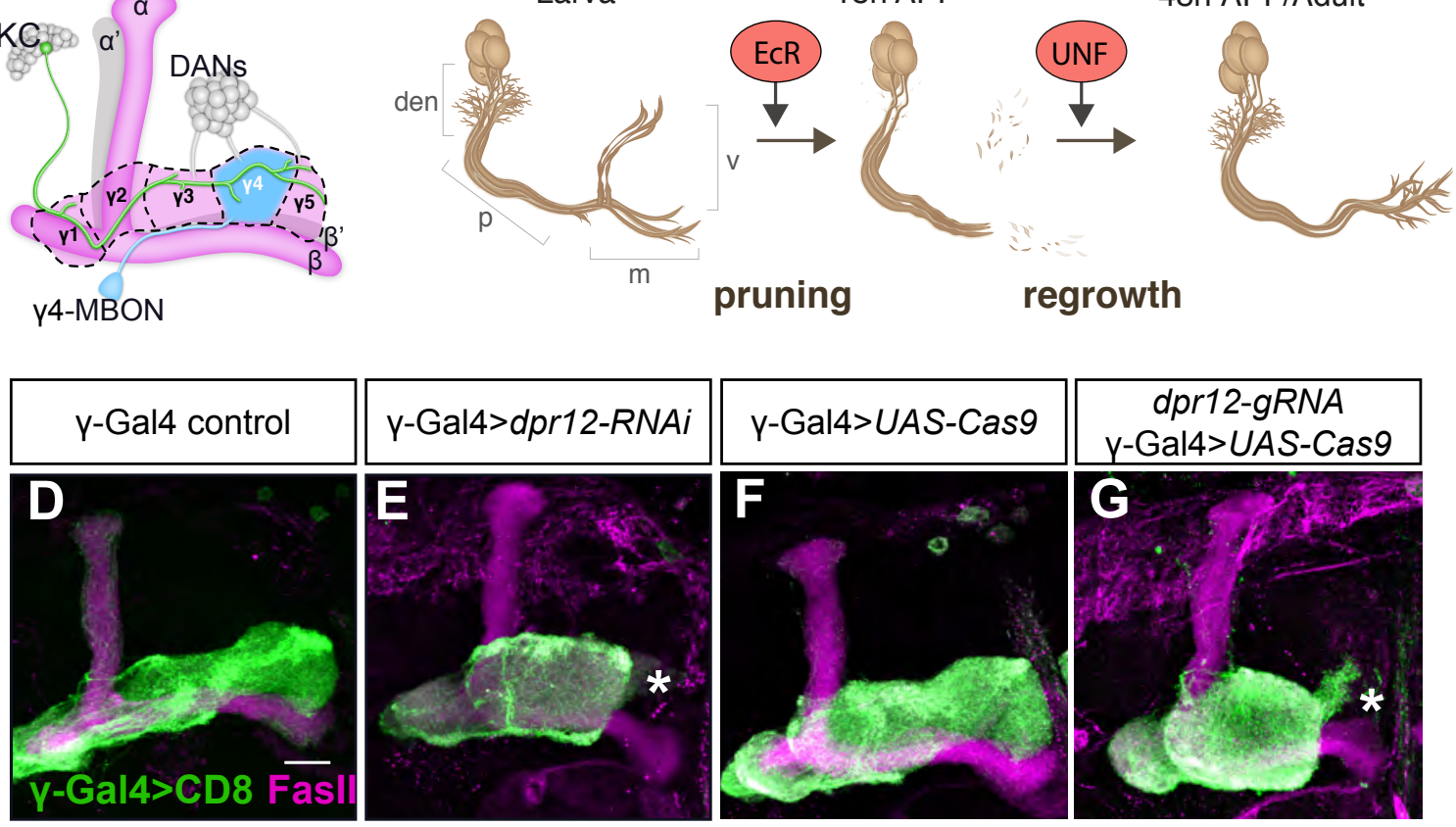

dpr12-gRNA Y-Gal4>UAS-Cas9

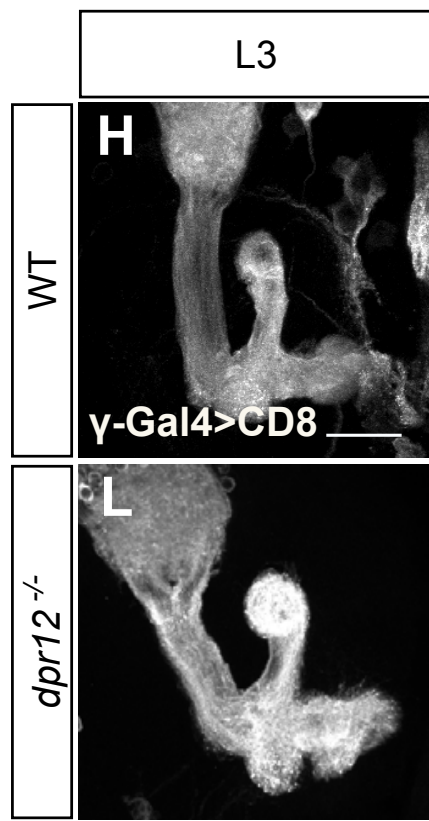

48hr APF

Adult

C
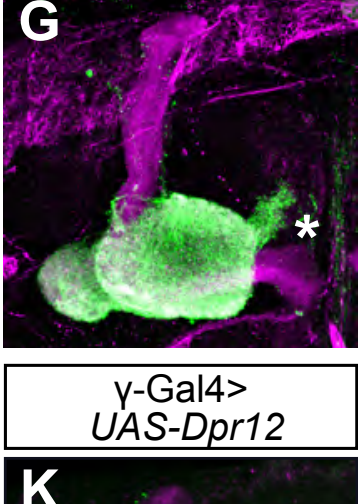

$\mathbf{K}$

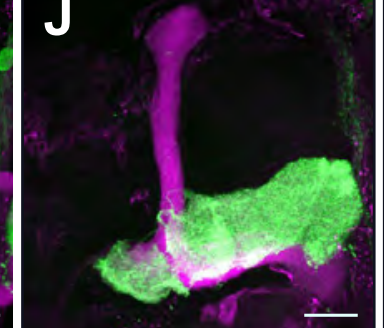

$\mathbf{N}$

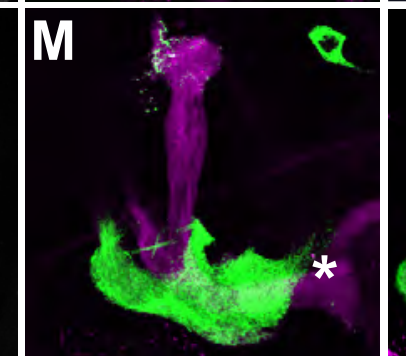

C Developmental expression

, hours after puparium formation,

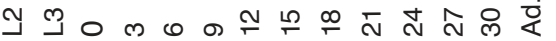
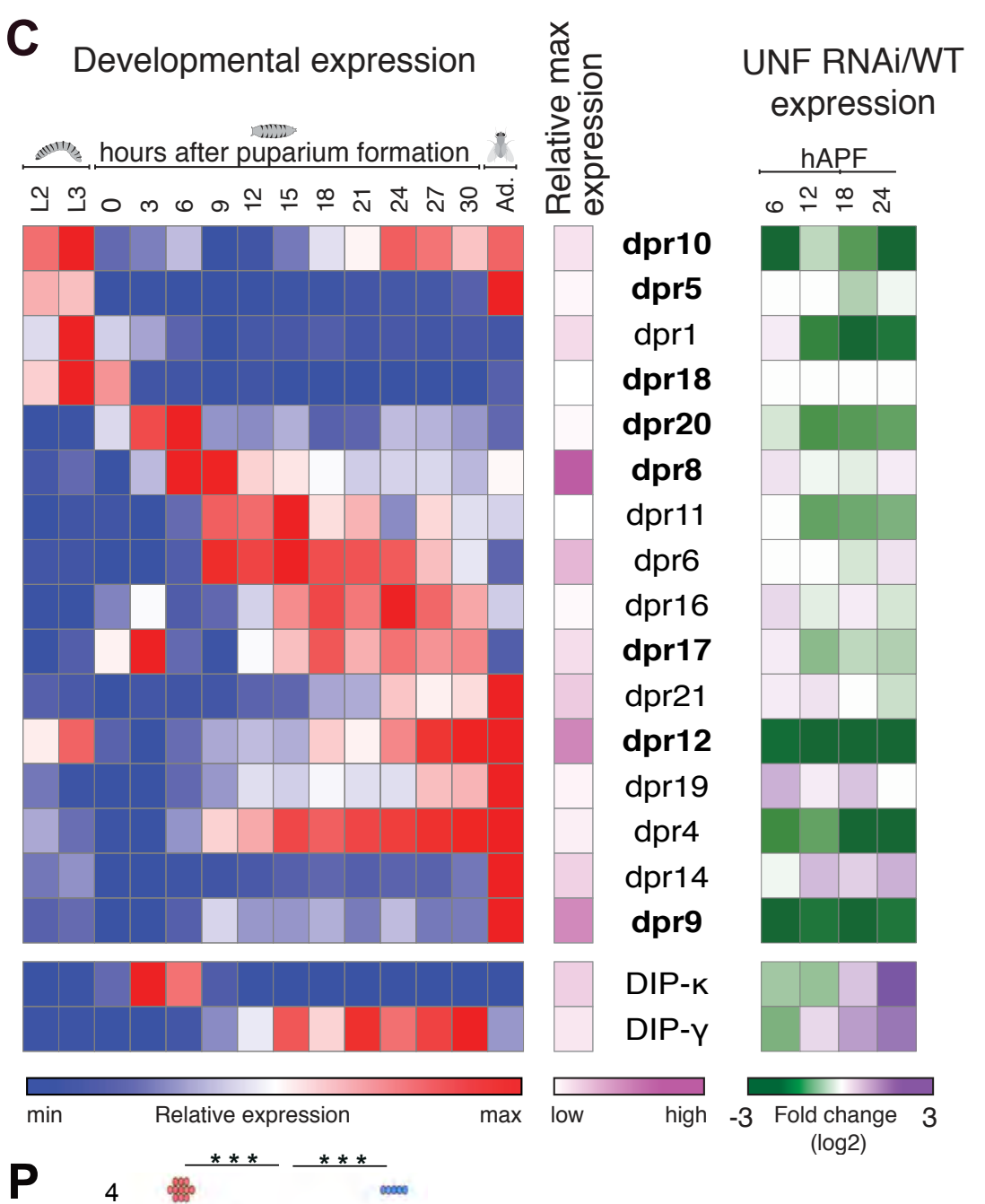

expression

$\frac{\text { hAPF }}{\underset{\infty}{\infty} \stackrel{\infty}{\sim}}$

dpr10

dpr5

dpr1

dpr18

dpr20

dpr8

dpr11

dpr6

dpr16

dpr17

dpr21

dpr12

dpr19

dpr4

dpr14

dpr9

DIP-K

DIP-Y

high -3 Fold change 3

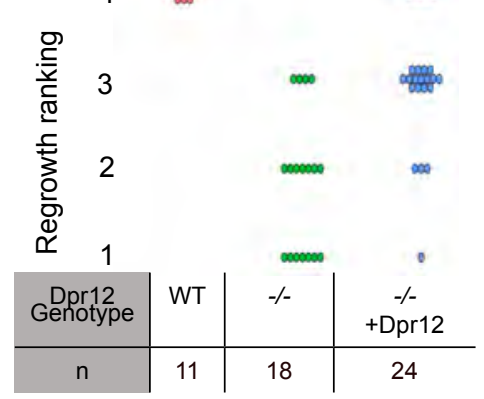




\section{Figure 1. Dpr12 is required for full extension of $\mathbf{Y - K C s .}$}

(A) Schematic representation of the adult MB. The bundled $y$-axons (an example of which is depicted in green) form the lobe. Stereotyped and tiled innervation of the lobe by Dopaminergic (DANs, gray) and MB output neurons (MBONs, an example of a $\mathrm{y} 4 \mathrm{MBON}$ is shown in cyan) define the $\mathrm{\gamma} 1-\mathrm{\gamma} 5$ zones.

400 (B) Schematic representation of neuron remodeling of $y-K C s$ and its regulation by the nuclear receptors EcR and UNF. den: dendrites; $p$ : axon peduncle; m/v: medial and vertical lobes.

(C) Dynamic expression of Dpr and DIPs during Y-KCs development. Left: Heatmap depicting the relative expression patterns of Dprs and DIPs in $\mathrm{Y}$-KCs during

405 development. Middle: Magenta intensity depicts the peak expression of each gene during development relative to other Dprs and DIPs. Right: Expression change of Dprs and DIPs while knocking down the UNF transcription factor compared to WT YKCs. Dprs highlighted in bold were tested in the RNAi mini-screen (Figure S1).

(D-O) Confocal z-projections of the indicated genotypes and age, labeled with 410 membrane bound GFP (mCD8-GFP; CD8) driven by the y specific Gal4 driver GMR71G10-Gal4 ( $\gamma$-Gal4). While $\gamma$-axons of control flies project through the entire lobe ( $D ; n=12 / 12, F ; n=14 / 14)$, knockdown of $d p r 12$ by RNAi $(E ; n=12 / 12)$ or knockout by tsCRISPR ( $\mathrm{G} ; \mathrm{n}=14 / 14$ ) resulted in short axons. At $L 3, \mathrm{Y}$-axons in $d p r 12^{\Delta 50-81}$ homozygote mutant animals $(\mathrm{L} ; \mathrm{n}=20 / 20)$ resemble WT $\mathrm{Y}$-axons $(\mathrm{H}$;

$415 n=20 / 20)$. At 48h APF, $Y$-axons normally $(I ; n=12 / 12)$ re-extend to form the adult lobe. $d p r 12^{\Delta 50-81} \mathrm{y}$-axons $(\mathrm{M} ; \mathrm{n}=14 / 14)$ fail to extend to the end of the lobe. This defect persists to adult $(\mathrm{J} ; \mathrm{n}=11 / 11, \mathrm{~N} ; \mathrm{n}=18 / 18)$. Expressing a UAS-Dpr12 transgene within $\mathrm{y}-\mathrm{KCs}$ in $d p r 12^{\triangle 50-81}$ homozygote mutant animals rescued the axon regrowth defect $(O ; n=23 / 24, K ; n=14 / 14)$.

420 (P) Quantification of the regrowth defects in $\mathrm{J}, \mathrm{N}$ and $\mathrm{O}$. The z-projections were blindly classified into four classes of regrowth defect severity, see Figure S1D for examples. Significance was calculated by Kruskal-Wallis test followed by a MannWhitney post-hoc test; ${ }^{* * *} p<0.001$.

Asterisks demarcate the distal part of the lobe. Green and white indicate mCD8GFP. Magenta represents Fasll staining. Scale bar is $20 \mu \mathrm{m}$. 
Bornstein et al. Figure 2
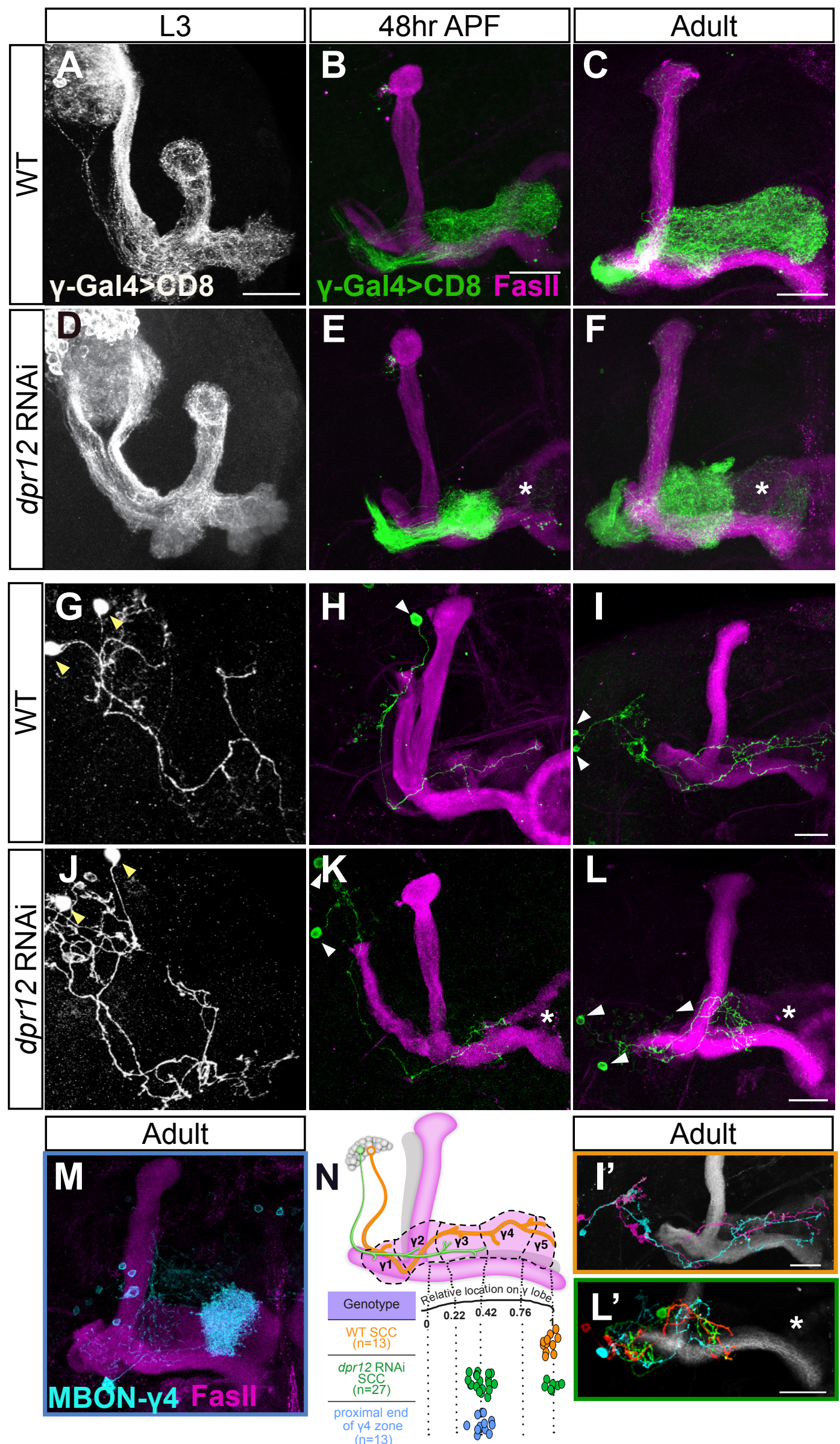
Figure 2. Dpr12 is cell autonomously required for $\mathrm{Y}$-axon regrowth into the $\mathrm{Y} 4 / 5$ zones.

430 (A-L) Confocal z-projections of MARCM neuroblast (NB, A-F) and single-cell (SC, G$\mathrm{L}$ ) clones labeled with membrane bound GFP (mCD8-GFP; CD8) driven by the $Y$ specific Gal4 driver GMR71G10-Gal4 ( $\gamma-$ Gal4). At L3, NB and SC clones expressing dpr12 RNAi are similar to equivalent WT clones (A; n=20/20, D; n=15/15, G; n=15/15 and J; $n=17 / 17)$. At 48hr APF and adult stage, WT NB (B; $n=15 / 15, C ; n=10 / 10)$ and $435 \mathrm{SC}(\mathrm{H} ; \mathrm{n}=16 / 16, \mathrm{I} ; \mathrm{n}=13 / 13)$ clones extend their axons to form the full adult lobe. In contrast, clones expressing dpr12 RNAi (E; n=14/14, F; n=22/2, K; n=18/24, L; $n=19 / 27$ ) fail to extend their axons to the distal part of the medial lobe (asterisks). l' and L' are traces of the single cell clones depicting each cell in a different color.

(M) Confocal z-projection of MBONy4>y1y2 labeled by GMR18H09-Gal4 driving the 440 expression of $m C D 8-G F P$ (CD8) shown in cyan.

(N) Top: Schematic representation of WT (orange) and dpr12 RNAi (green) expressing single $\mathrm{y}-\mathrm{KC}$ clones. Bottom: Measurements of the relative location to which WT (I) and dpr12 RNAi (L) axons grow across the entire length of the adult $Y$ lobe alongside the relative position of the proximal end of $\mathrm{Y} 4$ zone $(\mathrm{M}$, see also

445 Figure S2).

Arrowheads demarcate single cell bodies. Green, white and cyan represent mCD8GFP. Magenta shows Fasll. Scale bar is $20 \mu \mathrm{m}$. 
Bornstein et al. Figure 3

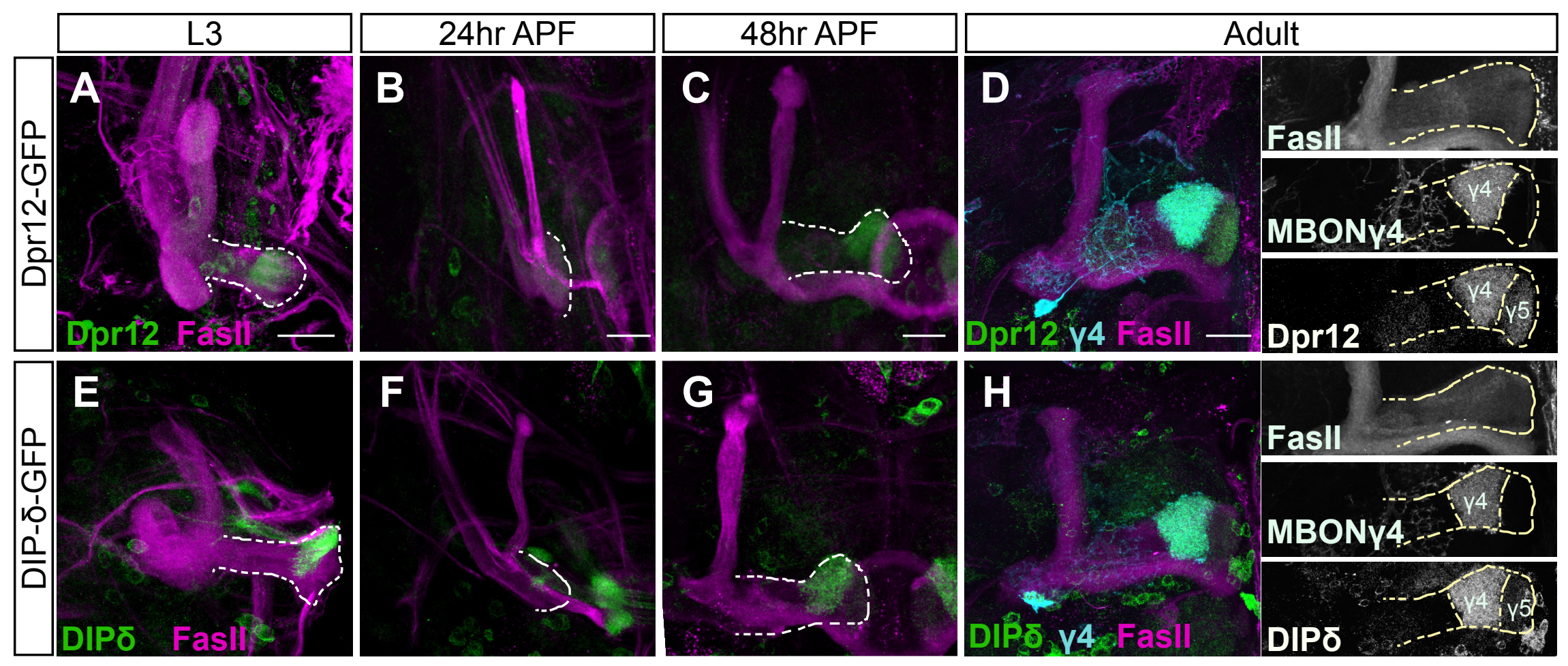


450 Figure 3. Both Dpr12 and its interacting protein DIP- $\delta$ localize to the $\mathrm{Y} 4 / 5$ zones.

(A-H) Confocal z-projections of brains expressing MiMIC mediated Dpr12 ${ }^{\text {GFSTF }}$ (Dpr12-GFP) and DIP- $\delta$ GFSTF (DIP- $\delta-G F P)$ fusion proteins at the indicated time points. See Figures S1 and S3 for more details on the fusion protein structure.

455 (A-D) Dpr12-GFP is localized to the distal part of the $\mathrm{y}$-lobe at $L 3(A ; n=10 / 10), 48 \mathrm{hr}$ APF (C; $n=12 / 12)$ and the adult stage ( $D ; n=20 / 20)$, where it colocalizes with a $\mathrm{Y} 4$ MBON ( $\mathrm{4}$; labeled by GMR18H09-Gal4 driving the expression of CD4-tdT). At 24hr APF (B; $n=10 / 10)$, Dpr12-GFP appears diffuse.

(E-H) DIP- $\delta-G F P$ is localized to the distal part of the $y$-lobe throughout development:

460 L3 (E; n=16/16), 24hr APF (F; n=10/10), 48hr APF (G; $n=12 / 12)$ and adult $(H$; $n=24 / 24)$. At the adult stage, DIP- $\delta-G F P$ is colocalized with a $\gamma 4 \mathrm{MBON}(\gamma 4)$.

Dashed line depicts the medial Y-lobe. Green is GFP, cyan is CD4-tdT, magenta is Fasll. Grayscale in right panels of $\mathrm{D}$ and $\mathrm{H}$ represent single channels, as marked. Scale bar is $20 \mu \mathrm{m}$. 
Bornstein et al. Figure 4

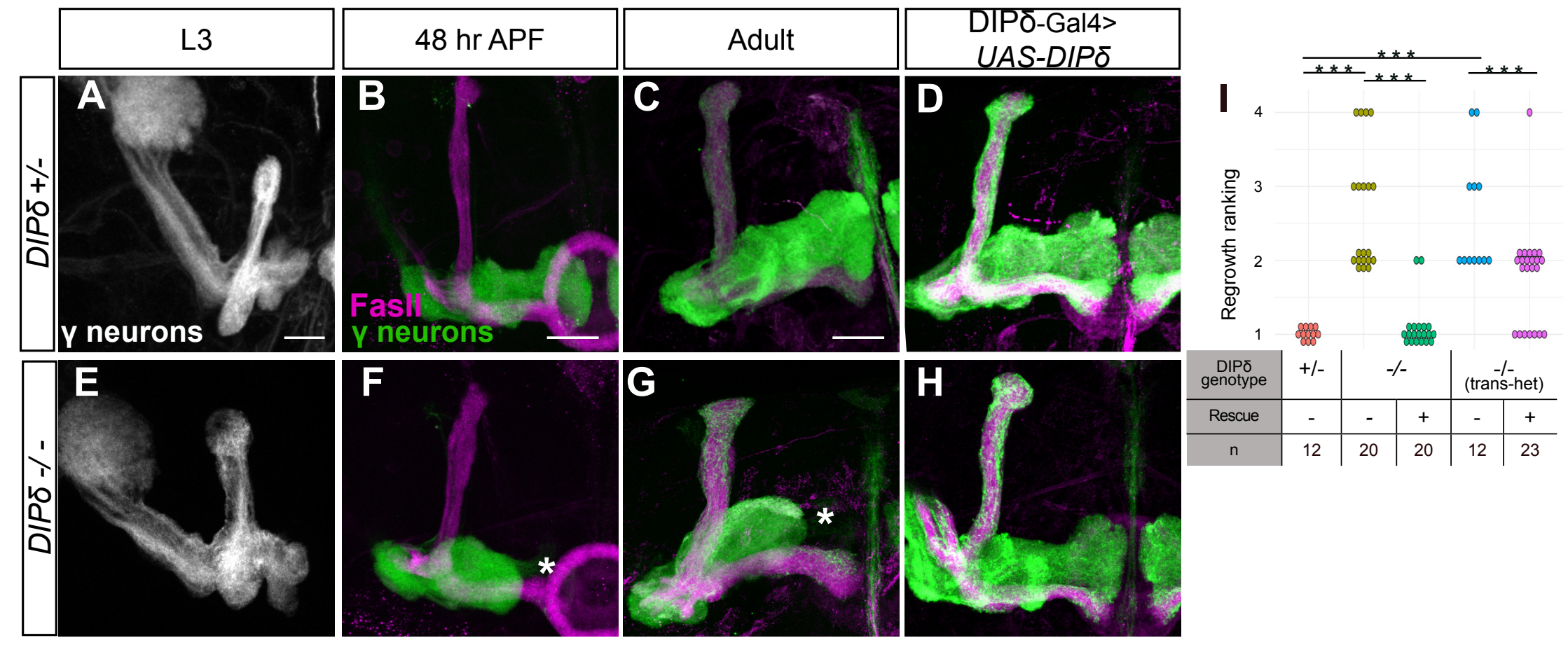




\section{Figure 4. DIP-ס is required for $\mathrm{y}$-axon regrowth into the $\mathrm{y} 4 / 5$ zones}

(A-H) Confocal z-projections DIP- $\delta$ hetero- and homozygous brains in which $\mathrm{Y}-\mathrm{KCs}$ were labeled by expressing membrane bound tandem tomato (mtdT-HA) driven by the $y$ specific QF2 driver R71G10-QF2 ( $\gamma$-neurons). Larval (L3) Y-axons grow normally in DIP- $\delta^{T 2 A-G a l 4}$ heterozygotes $(A ; n=16 / 16)$ and homozygotes $(E ; n=24 / 24)$. In contrast, at 48hr APF and adult, $\mathrm{Y}$-axons within DIP- $\delta^{T 2 A-G a l 4}$ homozygotes do not enter the distal part of the lobe (asterisks; F; $n=8 / 8, G ; n=20 / 20$ ), while they grow normally in heterozygotes $(B ; n=12 / 12, C ; n=12 / 12)$. Overexpression of a $D I P-\delta$

475 transgene driven by the Gal4 activity of $D I P-\delta^{T 2 A-G a l 4}$ (see also Figure S3) does not affect normal growth $(D ; n=10 / 10)$ and rescues mutant phenotypes $(H ; n=20 / 20)$. Asterisks demarcate distal part of the lobe. Green and white are mtdT-HA, magenta is Fasll, Scale bar is $20 \mu \mathrm{m}$.

(I) Quantification of the regrowth defects in C, D, G, H, and Figure S3C-E. Regrowth defect severity and statistics were calculated as in Fig. $1 ;{ }^{* *} p<0.001$ 
Bornstein et al. Figure 5
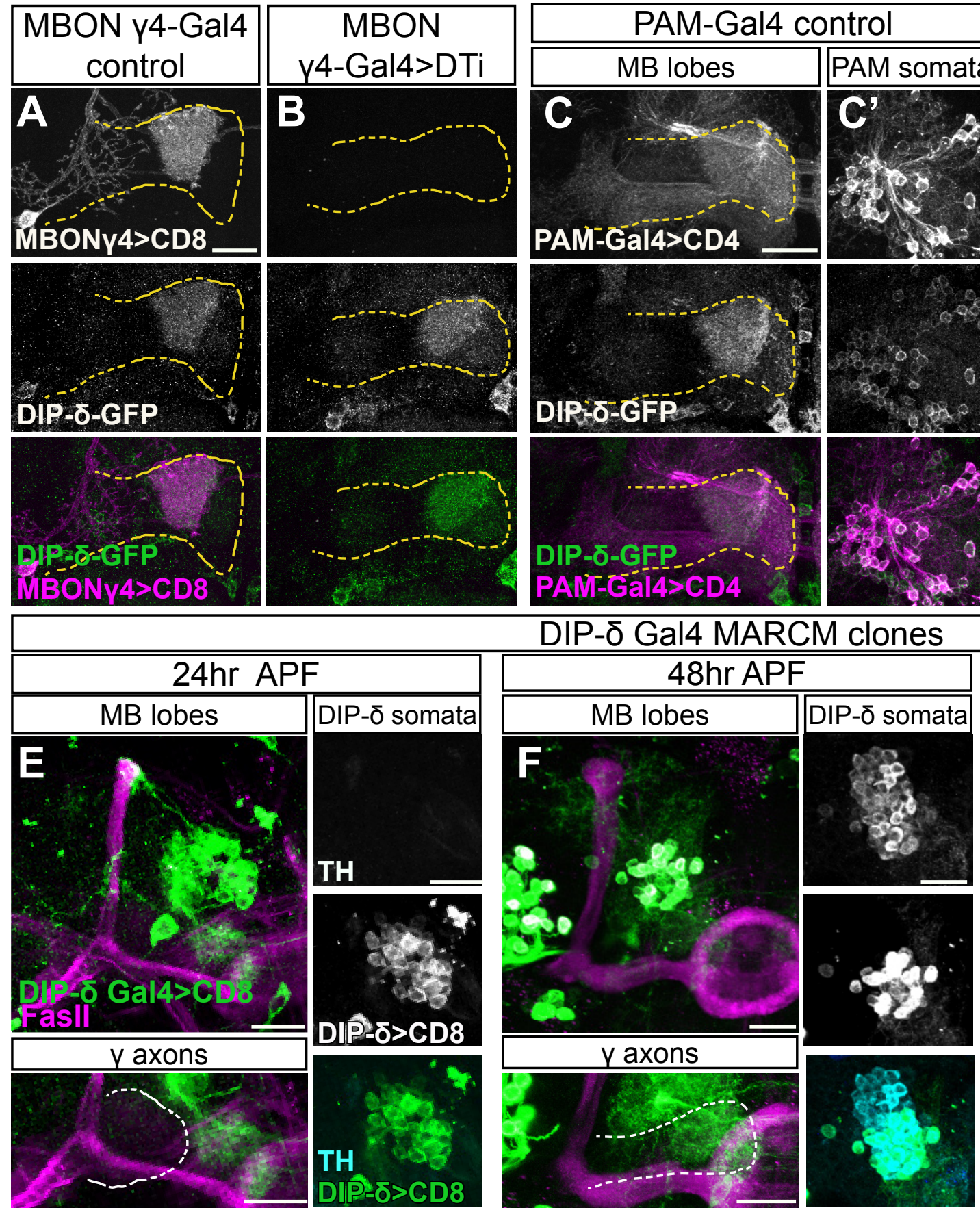
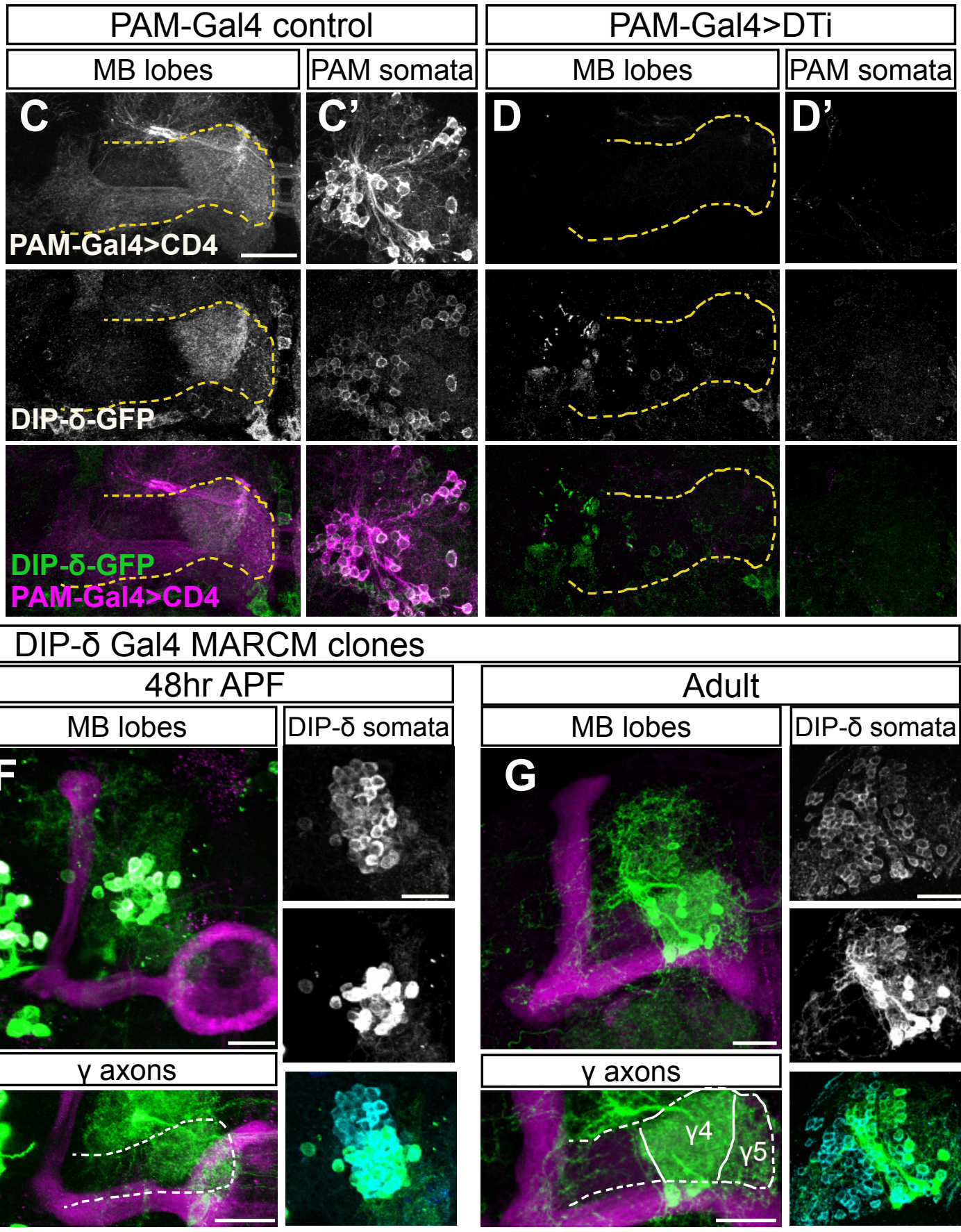
Figure 5. PAM DANs are the source of DIP- $\delta$ in the $y 4 / 5$ zones.

485 (A-D) Confocal z-projections of brains expressing DIP- $\delta$ GFSTF (DIP- $\delta$-GFP) together with the indicated $\mathrm{Gal} 4 \mathrm{~s}$ and transgenes. Expressing diphtheria toxin (DTi) and the membrane bound RFP (mCD8-RFP; CD8) driven by the 44 MBON driver MB294BGal4 (MBONy4-Gal4) did not affect DIP-ס-GFP expression (A, n=16/16; B, n=14/14). In contrast, similar expression of DTi and membrane bound Tomato (CD4-tdT; CD4)

490 in PAM-DANs (using the GMR58E02-Gal4; PAM-Gal4) abolished the normal DIP-סGFP expression in the $\mathrm{Y} 4 / 5$ zone (compare $D, n=18 / 18$, to $C, n=16 / 16$ ) and within the PAM cell bodies (compare D' to C').

Magenta is CD8-RFP (A-B), CD4-mtdT (C-D). Green is GFP, grayscale depict individual channels as labeled. Scale bar is $20 \mu \mathrm{m}$.

495 (E-G) Confocal z-projections of MARCM clones labeled by DIP- $\delta^{T 2 A-G a l 4}$ (DIP- $\delta$ Gal4) driving the expression of membrane bound GFP (mCD8-GFP; CD8) and heat shocked at $24 \mathrm{hr}$ after egg laying. Clones innervate the $\mathrm{y} 4 / 5$ zones at $24 \mathrm{hr}$ APF (E; $\mathrm{n}=8)$, 48hr APF ( $F ; n=8)$ and adult $(\mathrm{G} ; \mathrm{n}=16)$. Clones become tyrosine hydroxylase (TH) positive only at 48hr APF onwards $(F, G)$.

500 Magenta is Fasll, green is mCD8-GFP, cyan is TH antibody staining, grayscale single channels are shown as indicated. Scale bar is $20 \mu \mathrm{m}$. 
Bornstein et al. Figure 6
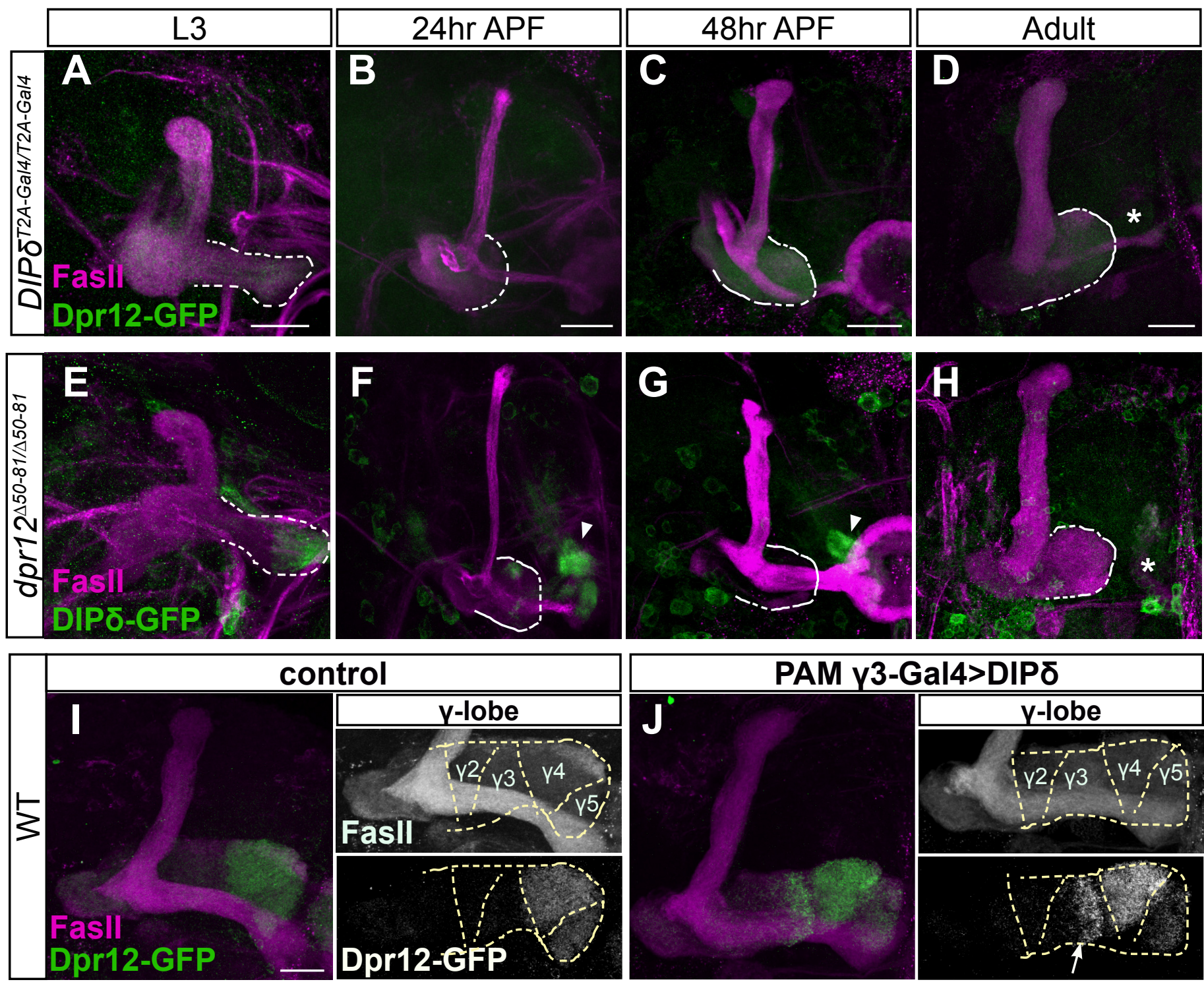


\section{Figure 6. DIP- $\delta$ is required and sufficient for Dpr12 localization}

(A-J) Confocal Z-projections of brains expressing MiMIC mediated Dpr12 GFSTF (Dpr12-GFP) and DIP- $\delta$ GFSTF (DIP- $\delta-G F P)$ fusion proteins at the indicated genotypes time points.

(A-D) Dpr12-GFP expression is diffuse in DIP- $\delta^{T 2 A-G a / 4}$ homozygotes mutant brains at L3 (A; $n=20 / 20), 24 h r$ APF (B; $n=14 / 14)$, 48hr APF (C; $n=28 / 28)$ and adult (D; $n=26 / 26)$.

510 (E-H) DIP-ס-GFP expression in $d p r 12^{\triangle 50-81}$ homozygotes mutant brains remains localized to the distal part of the $y$-lobe at $L 3(E ; n=16 / 16), 24 h r$ APF (F; $n=16 / 16)$, and 48hr APF (G; $n=10 / 10)$ but cannot be identified in adult brains $(H ; n=16 / 16)$.

(I-J) Dpr12-GFP expression in WT animals $(\mathrm{I}, \mathrm{n}=8 / 8)$ or in those ectopically expressing DIP- $\delta$ in PAM-DANs that innervate the $\gamma 3$ zone $(\mathrm{J}, \mathrm{n}=14 / 14)$ driven by

515 MB441B-Gal4 (PAMy3-Gal4). DIP- $\delta$ expression in PAMү3 resulted in Dpr12-GFP localization within the $\mathrm{\gamma} 3$ zone (arrow), in addition to its normal $\mathrm{\gamma} 4 / \mathrm{\gamma} 5$ localization.

Arrowheads demarcate DIP- $\delta$ expression at the distal part of the lobe. Asterisks demarcate the distal part of the lobe. Dashed line depicts the medial $y$-lobe, as determined by Fasll staining. Green is GFP, magenta is Fasll. Grayscale in right panels of I and $\mathrm{J}$ represent single channels, as marked. Scale bar is $20 \mu \mathrm{m}$. 
Bornstein et al. Figure 7
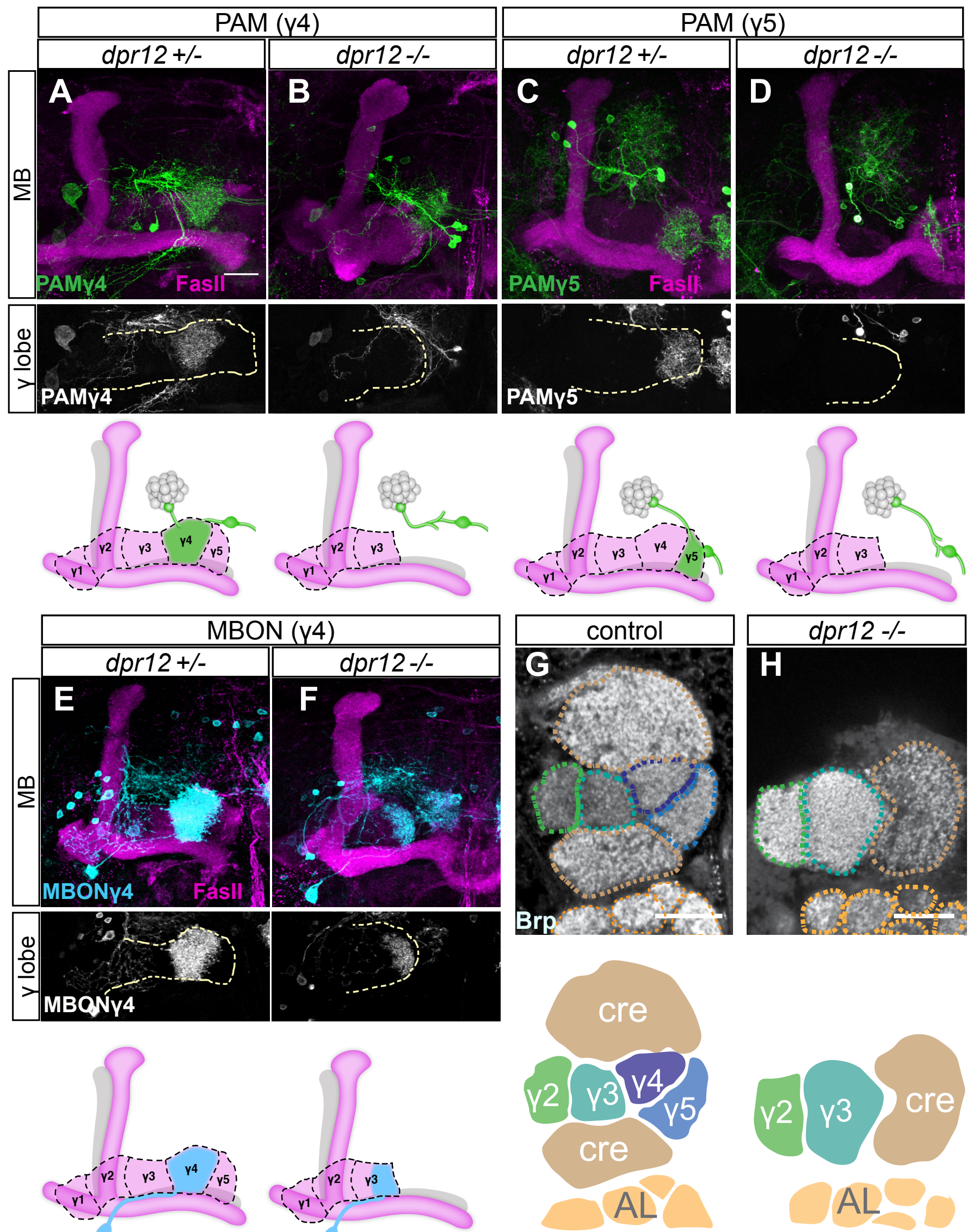


\section{Figure 7. Dpr12-DIP-ס interaction mediates circuit assembly}

(A-F) Top: Confocal z-projections of $d p r 12^{\Delta 50-81}$ heterozygous $(A, n=15 ; C, n=10 ; E$,

$525 \mathrm{n}=10)$ and homozygous brains $(\mathrm{B}, \mathrm{n}=8 ; \mathrm{D}, \mathrm{n}=18 ; \mathrm{F}, \mathrm{n}=24)$ expressing mCD8-GFP (CD8) driven by: (A-B) R10G03-Gal4 (PAMy4-Gal4); (C-D) R48H11-Gal4 (PAMy5Gal4), or (E-F) R18H09-Gal4 (MBONy4-Gal4). Bottom: Cartoons schematizing MB lobe structure and innervation by specific PAM-DANs or MBON.

(G-H) Single confocal slices of WT $(\mathrm{G}, \mathrm{n}=5 / 5)$ and $d p r 12^{\Delta 50-81}$ homozygous brains $(\mathrm{H}$, $530 \mathrm{n}=5 / 5)$ stained with anti-Brp. Dashed lines demarcate neuropil boundaries, schematic shown below. cre, crepin; AL, antenna lobe.

Magenta is Fasll, green and cyan are mCD8-GFP, grayscale depicts single channels as indicated. Scale bar is $20 \mu \mathrm{m}$. 


\section{References:}

Alyagor, I., Berkun, V., Keren-Shaul, H., Marmor-Kollet, N., David, E., Mayseless, O., Issman-Zecharya, N., Amit, I., and Schuldiner, O. (2018). Combining Developmental and Perturbation-Seq Uncovers Transcriptional Modules Orchestrating Neuronal Remodeling. Developmental cell 47, 38-52 e36.

540 Ashley, J., Sorrentino, V., Lobb-Rabe, M., Nagarkar-Jaiswal, S., Tan, L., Xu, S., Xiao, Q., Zinn, K., and Carrillo, R.A. (2019). Transsynaptic interactions between IgSF proteins DIP-alpha and Dpr10 are required for motor neuron targeting specificity. eLife 8.

Aso, Y., Hattori, D., Yu, Y., Johnston, R.M., Iyer, N.A., Ngo, T.T., Dionne, H., Abbott,

545 L.F., Axel, R., Tanimoto, H., et al. (2014a). The neuronal architecture of the mushroom body provides a logic for associative learning. eLife 3, e04577.

Aso, Y., Sitaraman, D., Ichinose, T., Kaun, K.R., Vogt, K., Belliart-Guerin, G., Placais, P.Y., Robie, A.A., Yamagata, N., Schnaitmann, C., et al. (2014b). Mushroom body output neurons encode valence and guide memory-based action

550 selection in Drosophila. eLife 3, e04580.

Bilz, F., Geurten, B.R.H., Hancock, C.E., Widmann, A., and Fiala, A. (2020). Visualization of a Distributed Synaptic Memory Code in the Drosophila Brain. Neuron.

Carrillo, R.A., Ozkan, E., Menon, K.P., Nagarkar-Jaiswal, S., Lee, P.T., Jeon, M.,

555 Birnbaum, M.E., Bellen, H.J., Garcia, K.C., and Zinn, K. (2015). Control of Synaptic Connectivity by a Network of Drosophila IgSF Cell Surface Proteins. Cell 163, 17701782.

Cognigni, P., Felsenberg, J., and Waddell, S. (2018). Do the right thing: neural network mechanisms of memory formation, expression and update in Drosophila.

560 Current opinion in neurobiology 49, 51-58.

Cohn, R., Morantte, I., and Ruta, V. (2015). Coordinated and Compartmentalized Neuromodulation Shapes Sensory Processing in Drosophila. Cell 163, 1742-1755.

Cosmanescu, F., Katsamba, P.S., Sergeeva, A.P., Ahlsen, G., Patel, S.D., Brewer, J.J., Tan, L., Xu, S., Xiao, Q., Nagarkar-Jaiswal, S., et al. (2018). Neuron-Subtype-

565 Specific Expression, Interaction Affinities, and Specificity Determinants of DIP/Dpr Cell Recognition Proteins. Neuron 100, 1385-1400 e1386.

Crittenden, J.R., Skoulakis, E.M., Han, K.A., Kalderon, D., and Davis, R.L. (1998). Tripartite mushroom body architecture revealed by antigenic markers. Learning \& memory 5, 38-51.

570 Croset, V., Treiber, C.D., and Waddell, S. (2018). Cellular diversity in the Drosophila midbrain revealed by single-cell transcriptomics. eLife 7.

Fiala, A. (2007). Olfaction and olfactory learning in Drosophila: recent progress. Current opinion in neurobiology 17, 720-726. 
Gerber, B., Scherer, S., Neuser, K., Michels, B., Hendel, T., Stocker, R.F., and Heisenberg, M. (2004). Visual learning in individually assayed Drosophila larvae. J Exp Biol 207, 179-188.

Heisenberg, M. (2003). Mushroom body memoir: from maps to models. Nature reviews Neuroscience 4, 266-275.

Lee, T., Lee, A., and Luo, L. (1999). Development of the Drosophila mushroom bodies: sequential generation of three distinct types of neurons from a neuroblast. Development 126, 4065-4076.

Lee, T., Marticke, S., Sung, C., Robinow, S., and Luo, L. (2000). Cell-autonomous requirement of the USP/EcR-B ecdysone receptor for mushroom body neuronal remodeling in Drosophila. Neuron 28, 807-818.

585 Meltzer, H., Marom, E., Alyagor, I., Mayseless, O., Berkun, V., Segal-Gilboa, N., Unger, T., Luginbuhl, D., and Schuldiner, O. (2019). Tissue-specific (ts)CRISPR as an efficient strategy for in vivo screening in Drosophila. Nature communications 10 , 2113.

Modi, M.N., Shuai, Y., and Turner, G.C. (2020). The Drosophila Mushroom Body: 590 From Architecture to Algorithm in a Learning Circuit. Annual review of neuroscience.

Nagarkar-Jaiswal, S., Lee, P.T., Campbell, M.E., Chen, K., Anguiano-Zarate, S., Gutierrez, M.C., Busby, T., Lin, W.W., He, Y., Schulze, K.L., et al. (2015). A library of MiMICs allows tagging of genes and reversible, spatial and temporal knockdown of proteins in Drosophila. eLife 4.

595 Owald, D., and Waddell, S. (2015). Olfactory learning skews mushroom body output pathways to steer behavioral choice in Drosophila. Current opinion in neurobiology 35, 178-184.

Ozkan, E., Carrillo, R.A., Eastman, C.L., Weiszmann, R., Waghray, D., Johnson, K.G., Zinn, K., Celniker, S.E., and Garcia, K.C. (2013). An extracellular interactome of immunoglobulin and LRR proteins reveals receptor-ligand networks. Cell 154, 228-239.

Port, F., and Bullock, S.L. (2016). Augmenting CRISPR applications in Drosophila with tRNA-flanked sgRNAs. Nature methods 13, 852-854.

Port, F., Strein, C., Stricker, M., Rauscher, B., Heigwer, F., Zhou, J., Beyersdorffer, 605 C., Frei, J., Hess, A., Kern, K., et al. (2020). A large-scale resource for tissue-specific CRISPR mutagenesis in Drosophila. eLife 9.

Rabinovich, D., Yaniv, S.P., Alyagor, I., and Schuldiner, O. (2016). Nitric Oxide as a Switching Mechanism between Axon Degeneration and Regrowth during Developmental Remodeling. Cell 164, 170-182.

610 Rohwedder, A., Wenz, N.L., Stehle, B., Huser, A., Yamagata, N., Zlatic, M., Truman, J.W., Tanimoto, H., Saumweber, T., Gerber, B., et al. (2016). Four Individually 
Identified Paired Dopamine Neurons Signal Reward in Larval Drosophila. Current biology : CB 26, 661-669.

Sanz, R., Ferraro, G.B., and Fournier, A.E. (2015). IgLON cell adhesion molecules 615 are shed from the cell surface of cortical neurons to promote neuronal growth. The Journal of biological chemistry 290, 4330-4342.

Saumweber, T., Rohwedder, A., Schleyer, M., Eichler, K., Chen, Y.C., Aso, Y., Cardona, A., Eschbach, C., Kobler, O., Voigt, A., et al. (2018). Functional architecture of reward learning in mushroom body extrinsic neurons of larval 620 Drosophila. Nature communications 9, 1104.

Shuai, Y., Hirokawa, A., Ai, Y., Zhang, M., Li, W., and Zhong, Y. (2015). Dissecting neural pathways for forgetting in Drosophila olfactory aversive memory. Proceedings of the National Academy of Sciences of the United States of America 112, E66636672.

625 Tan, L., Zhang, K.X., Pecot, M.Y., Nagarkar-Jaiswal, S., Lee, P.T., Takemura, S.Y., McEwen, J.M., Nern, A., Xu, S., Tadros, W., et al. (2015). Ig Superfamily Ligand and Receptor Pairs Expressed in Synaptic Partners in Drosophila. Cell 163, 1756-1769.

Tanaka, N.K., Tanimoto, H., and Ito, K. (2008). Neuronal assemblies of the Drosophila mushroom body. J Comp Neurol 508, 711-755.

630 Venkatasubramanian, L., Guo, Z., Xu, S., Tan, L., Xiao, Q., Nagarkar-Jaiswal, S., and Mann, R.S. (2019). Stereotyped terminal axon branching of leg motor neurons mediated by IgSF proteins DIP-alpha and Dpr10. eLife 8.

Xu, S., Xiao, Q., Cosmanescu, F., Sergeeva, A.P., Yoo, J., Lin, Y., Katsamba, P.S., Ahlsen, G., Kaufman, J., Linaval, N.T., et al. (2018). Interactions between the Ig-

635 Superfamily Proteins DIP-alpha and Dpr6/10 Regulate Assembly of Neural Circuits. Neuron 100, 1369-1384 e1366.

Yaniv, S.P., Issman-Zecharya, N., Oren-Suissa, M., Podbilewicz, B., and Schuldiner, O. (2012). Axon regrowth during development and regeneration following injury share molecular mechanisms. Current biology : CB 22, 1774-1782.

640 Yaniv, S.P., Meltzer, H., Alyagor, I., and Schuldiner, O. (2020). Developmental axon regrowth and primary neuron sprouting utilize distinct actin elongation factors. The Journal of cell biology 219.

Zinn, K., and Ozkan, E. (2017). Neural immunoglobulin superfamily interaction networks. Current opinion in neurobiology 45, 99-105. 


\section{Supplemental materials and STAR methods}

\section{Transneuronal Dpr12/DIP-ס interactions facilitate compartmentalized dopaminergic innervation of Drosophila mushroom body axons}

Bavat Bornstein ${ }^{1}$, Idan Alyagor ${ }^{1}$, Victoria Berkun ${ }^{1}$, Hagar Meltzer ${ }^{1}$, Fabienne Reh², Hadas Keren-Shaul ${ }^{3,4}$, Eyal David ${ }^{3}$, Thomas Riemensperger ${ }^{2}$ and Oren Schuldiner ${ }^{1^{*}}$

Correspondence to: oren.schuldiner@weizmann.ac.il 


\section{KEY RESOURCES TABLE}

\begin{tabular}{|c|c|c|}
\hline REAGENT or RESOURCE & SOURCE & IDENTIFIER \\
\hline \multicolumn{3}{|l|}{ Antibodies } \\
\hline Chicken anti GFP 1:500 & AVES & $\begin{array}{l}\text { GFP-1020 } \\
\text { RRID: } A B \quad 10000240\end{array}$ \\
\hline Mouse monoclonal anti Fasll 1:25 & $\begin{array}{l}\text { Developmental } \\
\text { Studies Hybridoma } \\
\text { Bank (DSHB) }\end{array}$ & $\begin{array}{l}\text { 1D4 } \\
\text { RRID: AB } 528235\end{array}$ \\
\hline Mouse monoclonal anti Brp 1:5 & DSHB & $\begin{array}{l}\text { nc82 } \\
\text { RRID: AB } 2314866\end{array}$ \\
\hline Rabbit anti TH 1:500 & Merck Millipore & $\begin{array}{l}\text { AB152 } \\
\text { RRID: AB } 390204\end{array}$ \\
\hline Rat anti RFP 1:500 & ChromoTek & $\begin{array}{l}5 f 8 \\
\text { RRID: AB 2336064 }\end{array}$ \\
\hline Alexa fluor 568 Goat anti Rat 1:500 & Invitrogen & $\begin{array}{l}\text { A-21247 } \\
\text { RRID: } A B \quad 2534121\end{array}$ \\
\hline Alexa fluor 647 Goat anti Mouse 1:500 & Invitrogen & $\begin{array}{l}\text { A-32728 } \\
\text { RRID: AB } 2633277\end{array}$ \\
\hline Alexa fluor 488 Goat anti Mouse 1:500 & Invitrogen & $\begin{array}{l}\text { A-11001 } \\
\text { RRID: } A B \quad 2534069\end{array}$ \\
\hline FITC Goat anti Chicken 1:500 & Invitrogen & $\begin{array}{l}\text { A-16055 } \\
\text { RRID: AB 2534728 }\end{array}$ \\
\hline Alexa fluor 568 Goat anti Rabbit 1:500 & Invitrogen & $\begin{array}{l}\text { A-11036 } \\
\text { RRID: AB } 10563566 \\
\end{array}$ \\
\hline \multicolumn{3}{|l|}{ Bacterial and Virus Strains } \\
\hline \multicolumn{3}{|l|}{ DH5a } \\
\hline \multicolumn{3}{|l|}{ Chemicals, Peptides, and Recombinant Proteins } \\
\hline Cell Dissociation Solution & Sigma Aldrich & Cat\# C1544 \\
\hline Collagenase/Dispase mix & Roche & Cat\# 10269638001 \\
\hline poly-L-lysine & Sigma Aldrich & Cat\# P1524-25MG \\
\hline \multicolumn{3}{|l|}{ Critical Commercial Assays } \\
\hline Pico pure RNA isolation kit & Thermo Fisher & Cat\# KIT0204 \\
\hline Gibson assembly & NEB & Cat\# E5510S \\
\hline \multicolumn{3}{|l|}{ Deposited Data } \\
\hline Raw data files for UNF Perturbation seq & $\begin{array}{l}\text { Will be deposited in } \\
\text { the NCBI Gene } \\
\text { Expression Omnibus }\end{array}$ & \\
\hline \multicolumn{3}{|l|}{ Experimental Models: D. melanogaster } \\
\hline $\begin{array}{l}w[1118] ; P\{y[+t 7.7] w[+m C]=G M R 71 G 10-G A L 4\} a t t P 2 \\
\text { (R71G10-Gal4) }\end{array}$ & $\begin{array}{l}\text { Bloomington } \\
\text { Drosophila Stock } \\
\text { Center (BDSC) }\end{array}$ & $\begin{array}{l}\text { BDSC: } 39604 \\
\text { FlyBase ID (FBID): } \\
\text { FBsf0000166728 }\end{array}$ \\
\hline $\mathrm{y}^{*}, \mathrm{w}^{*} ; P\{y[+t 7.7] w[+m C]=G M R 71 G 10-G A L 4\}$ attP40 & (Alyagor et al., 2018) & N/A \\
\hline $\mathrm{P}\{$ GawB\}elav 155 (C155-GAL4) & BDSC & $\begin{array}{l}\text { BDSC: } 458 \\
\text { FBID: FBti0002575 }\end{array}$ \\
\hline w $^{1118 ;}$ P\{GAL4\}repo/TM3, Sb1 (Repo-Gal4) & BDSC & $\begin{array}{l}\text { BDSC: } 7415 \\
\text { FBID: FBti0018692 }\end{array}$ \\
\hline $\begin{array}{l}w[1118] ; P\{y[+t 7.7] w[+m C]=G M R 58 E 02-G A L 4\} a t t P 2 \\
\text { (R58E02-Gal4) }\end{array}$ & BDSC & $\begin{array}{l}\text { BDSC: } 41347 \\
\text { FBID: FBtp0061564 }\end{array}$ \\
\hline $\begin{array}{l}w[1118] ; P\{y[+t 7.7] w[+m C]=G M R 18 H 09-G A L 4\} a t t P 2 \\
\text { (R18H09-Gal4) }\end{array}$ & BDSC & $\begin{array}{l}\text { BDSC: } 48830 \\
\text { FBID: FBti0133650 }\end{array}$ \\
\hline
\end{tabular}




\begin{tabular}{|c|c|c|}
\hline $\begin{array}{l}w[1118] ; P\{y[+t 7.7] w[+m C]=G M R 48 H 11-G A L 4\} a t t P 2 \\
\text { (R48H11-GAL4) }\end{array}$ & BDSC & $\begin{array}{l}\text { BDSC: } 50396 \\
\text { FBID: FBti0136291 }\end{array}$ \\
\hline $\begin{array}{l}w[1118] ; P\{y[+t 7.7] w[+m C]=G M R 10 G 03-G A L 4\} a t t P 2 \\
\text { (R10G03-Gal4) }\end{array}$ & BDSC & $\begin{array}{l}\text { BDSC: } 48271 \\
\text { FBID: FBti0132904 }\end{array}$ \\
\hline $\begin{array}{l}P\left\{y[+t 7.7] w[+m C]=G M R 71 G 10-Q F 2^{H S P}\right\} \text { attP40 } \\
(R 71 G 10-Q F 2)\end{array}$ & This study & N/A \\
\hline $\begin{array}{l}y[1] w[*] ; P B a c\{y[+m D i n t 2] w[+m C]=10 X Q U A S- \\
6 X G F P\} V K 00018, P\{w[+m C]=U A S-m t d T o m a t o-3 x H A\} 2 ; \\
P\{y[+t 7.7] w[+m C]=G M R 58 E 02-Q F 2 . L\} a t t P 2 \text { (R58E02- } \\
\text { QF2) }\end{array}$ & BDSC & $\begin{array}{l}\text { BDSC: } 66480 \\
\text { FBID: FBti0184753 }\end{array}$ \\
\hline $\begin{array}{l}w[1118] ; P\{y[+t 7.7] w[+m C]=R 53 C 03-p 65 . A D\} a t t P 40 ; \\
P\{y[+t 7.7] w[+m C]=R 24 E 12-G A L 4 . D B D\} a t t P 2 / T M 6 B \\
T b[1] \text { (MB298B-Gal4) }\end{array}$ & BDSC & $\begin{array}{l}\text { BDSC: } 68309 \\
\text { FBID: FBst0068309 }\end{array}$ \\
\hline $\begin{array}{l}w[1118] ; P\{y[+t 7.7] w[+m C]=R 30 G 08-p 65 . A D\} \text { attP40; } \\
P\{y[+t 7.7] w[+m C]=R 48 B 03-G A L 4 . D B D\} a t t P 2 \text { (MB441B- } \\
\text { Gal4) }\end{array}$ & BDSC & $\begin{array}{l}\text { BDSC: } 68251 \\
\text { FBID: FBst0068251 }\end{array}$ \\
\hline $\begin{array}{l}w[*] ; P\{w[+m W . h s]=G a w B\} O K 107 \text { ey[OK107] (OK107- } \\
\text { Gal4) }\end{array}$ & BDSC & $\begin{array}{l}\text { BDSC: } 854 \\
\text { FBID: FBti0004170 }\end{array}$ \\
\hline $\mathrm{y}^{*}, \mathrm{w}^{*} ; \mathrm{DIP}-\delta-\mathrm{T} 2 \mathrm{~A}-\mathrm{Gal} 4$ & This study & N/A \\
\hline $\begin{array}{l}w\left[^{*}\right] ; P\{y[+t 7.7] w[+m C]=10 X U A S-I V S-m C D 8:: G F P\} a t t P 2 \\
\text { (UAS-CD8-GFP) }\end{array}$ & BDSC & $\begin{array}{l}\text { BDSC: } 32185 \\
\text { FBID: FBst0032185 }\end{array}$ \\
\hline $\begin{array}{l}w[*] ; P\{y[+t 7.7] w[+m C]=10 X U A S-I V S-m C D 8:: G F P\} \\
a t t P 40 \text { (UAS-CD8-GFP) }\end{array}$ & BDSC & $\begin{array}{l}\text { BDSC: } 32186 \\
\text { FBID: FBst0032185 }\end{array}$ \\
\hline $\begin{array}{l}y[1] w\left[^{*}\right] P\{y[+t 7.7] w[+m C]=10 X U A S-I V S- \\
m C D 8:: G F P\} \text { su(Hw)attP8 (UAS-CD8-GFP) }\end{array}$ & BDSC & $\begin{array}{l}\text { BDSC: } 32189 \\
\text { FBID: FBst0032189: }\end{array}$ \\
\hline $\begin{array}{l}\left.y[1] w{ }^{*}\right] ; P\{w[+m C]=U A S-C D 4-t d T o m\} 7 M 1 \\
\text { (UAS-CD4-tdT) }\end{array}$ & BDSC & $\begin{array}{l}\text { BDSC: } 35841 \\
\text { FBID: FBst0035841 }\end{array}$ \\
\hline $\begin{array}{l}y[1] w[1118] ; P\{w[+m C]=Q U A S-m t d T o m a t o-3 x H A\} 26 \\
\text { (QUAS-mtdT) }\end{array}$ & BDSC & $\begin{array}{l}\text { BDSC: } 30005 \\
\text { FBID: FBti0129951 }\end{array}$ \\
\hline$w[1118] ; P\{w[+m C]=U A S-D c r-2 . D\} 10$ & BDSC & $\begin{array}{l}\text { BDSC: } 24651 \\
\text { FBID: FBst0024651 }\end{array}$ \\
\hline $\begin{array}{l}\mathrm{P}\{\mathrm{hsFLP}\} 22, \mathrm{w}^{*}, \mathrm{P}\{\mathrm{w}[+\mathrm{mC}]=\mathrm{UAS}-\mathrm{mCD} 8: \text { GFP.L }\} \\
\mathrm{P}\{\mathrm{w}[+\mathrm{mC}]=\text { tubP-GAL80\}LL10 P }\{\mathrm{ry}[+\mathrm{t} 7.2]=\text { neoFRT }\} 40 A ; \\
\text { GMR71G10-Gal4, P }\{\mathrm{w}[+\mathrm{mC}]=\mathrm{UAS}-\mathrm{Dcr}-2 . \mathrm{D}\} 10 \\
\text { (recombination between R71G10-Gal4 and UAS-Dcr-2) }\end{array}$ & This study & $\mathrm{N} / \mathrm{A}$ \\
\hline $\begin{array}{l}\text { P\{hsFLP }\} 22, w^{*}, P\{w[+m C]=U A S-m C D 8:: G F P . L\} \\
\text { P }\{w[+m C]=t u b P-G A L 80\} L L 10 \text { P }\{r y[+t 7.2]=\text { neoFRT }\} 40 A ; \\
\text { DIP- } \delta-T 2 A-G a l 4\end{array}$ & This study & $\mathrm{N} / \mathrm{A}$ \\
\hline $\begin{array}{l}\mathrm{y}[1] \mathrm{v}[1] ; \mathrm{P}\{\mathrm{y}[+\mathrm{t} 7.7] \mathrm{v}[+\mathrm{t} 1.8]=T R i P . J F 03306\} a t t P 2(\mathrm{RNAi} \\
\text { of Dpr5) }\end{array}$ & BDSC & $\begin{array}{l}\text { BDSC: } 29627 \\
\text { FBID: FBst0029627 }\end{array}$ \\
\hline $\begin{array}{l}y[1] v[1] ; P\{y[+t 7.7] v[+t 1.8]=T R i P . J F 03172\} a t t P 2(R N A i \\
\text { of Dpr8) }\end{array}$ & BDSC & $\begin{array}{l}\text { BDSC: } 28744 \\
\text { FBID: FBst0028744 }\end{array}$ \\
\hline $\begin{array}{l}y[1] \mathrm{sc}\left[{ }^{*}\right] v[1] ; \mathrm{P}\{\mathrm{y}[+\mathrm{t} 7.7] \mathrm{v}[+\mathrm{t} 1.8]=T R \mathrm{PiP} . \mathrm{HMS} 00288\} \text { attP2 } \\
\text { (RNAi of Dpr9) }\end{array}$ & BDSC & $\begin{array}{l}\text { BDSC: } 33409 \\
\text { FBID: FBst0033409 }\end{array}$ \\
\hline $\begin{array}{l}y[1] v[1] ; P\{y[+t 7.7] v[+t 1.8]=T R i P . J F 02920\} a t t P 2(R N A i \\
\text { of Dpr10) }\end{array}$ & BDSC & $\begin{array}{l}\text { BDSC: } 27991 \\
\text { FBID: FBst0027991 }\end{array}$ \\
\hline $\begin{array}{l}y[1] v[1] ; P\{y[+t 7.7] v[+t 1.8]=T R i P . J F 03210\} a t t P 2 \text { (RNAi } \\
\text { of Dpr12) }\end{array}$ & BDSC & $\begin{array}{l}\text { BDSC: } 28782 \\
\text { FBID: FBst0028782 }\end{array}$ \\
\hline $\begin{array}{l}\text { [1] v[1]; P }\{y[+t 7.7] v[+t 1.8]=T R i P . G L 01238\} a t t P 2(R N A i \\
\text { of Dpr17) }\end{array}$ & BDSC & $\begin{array}{l}\text { BDSC: } 41656 \\
\text { FBID: FBst0041656 }\end{array}$ \\
\hline $\begin{array}{l}\mathrm{y}[1] \mathrm{v}[1] ; \mathrm{P}\{\mathrm{y}[+\mathrm{t} 7.7] \mathrm{v}[+\mathrm{t} 1.8]=\mathrm{TRiP} . J F 03283\} a t t P 2(\mathrm{RNAi} \\
\text { of Dpr18) }\end{array}$ & BDSC & $\begin{array}{l}\text { BDSC: } 29604 \\
\text { FBID: FBst0029604 }\end{array}$ \\
\hline $\begin{array}{l}\text { [1] v[1]; P\{y[+t7.7] v[+t1.8]=TRiP.JF02923\}attP2 (RNAi } \\
\text { of Dpr20) }\end{array}$ & BDSC & $\begin{array}{l}\text { BDSC: } 28293 \\
\text { FBID: FBst0028293 }\end{array}$ \\
\hline
\end{tabular}




\begin{tabular}{|c|c|c|}
\hline $\begin{array}{l}P\{r y[+t 7.2]=h s F L P\} 12, y[1] w\left[{ }^{*}\right] ; P\{y[+t 7.7] \\
w[+m C]=U A S-C a s 9 . P 2\} \text { attP40 }\end{array}$ & BDSC & $\begin{array}{l}\text { BDSC: } 58985 \\
\text { FBID: FBst0058985 }\end{array}$ \\
\hline$w[1118] ; P\{y[+t 7.7] w[+m C]=U A S-C a s 9 . C\} a t t P 2$ & BDSC & $\begin{array}{l}\text { BDSC: } 54595 \\
\text { FBID:FBst0054595 }\end{array}$ \\
\hline gRNA dpr12 & This study & N/A \\
\hline 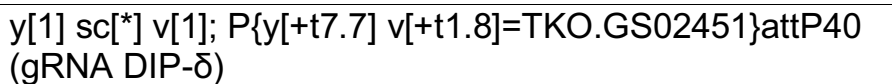 & BDSC & $\begin{array}{l}\text { BDSC: } 78754 \\
\text { FBID: FBst0078754 }\end{array}$ \\
\hline $\mathrm{y}^{*}, \mathrm{w}^{*} ; \mathrm{FRT} 40 \mathrm{~A}, \mathrm{dpr} 12^{\Delta 50-81}, \mathrm{FRTG} 13, \mathrm{cn}, \mathrm{bw}$ & This study & N/A \\
\hline $\mathrm{w}^{*} ;$ DIPס ${ }^{1-119}, \mathrm{FRT} 2 \mathrm{~A}$ & $\begin{array}{l}\text { A generous gift from } \\
\text { Larry Zipursky, UCLA }\end{array}$ & N/A \\
\hline $\begin{array}{l}\text { y[1] w[67c23]; Mi\{PT-GFSTF. 1\}dpr12[MI01695- } \\
\text { GFSTF.1]/SM6a (dpr12GFSTF) }\end{array}$ & BDSC & $\begin{array}{l}\text { BDSC: } 60171 \\
\text { FBID: FBst0060171 }\end{array}$ \\
\hline $\begin{array}{l}y[1] w\left[{ }^{*}\right] ; \text { Mi\{PT-GFSTF. 1\}DIP-delta[MI08287-GFSTF.1] } \\
\text { (DIP- } \delta^{\text {GFSTF})}\end{array}$ & BDSC & $\begin{array}{l}\text { BDSC: } 60558 \\
\text { FBID: FBst0060558 }\end{array}$ \\
\hline$\left.y[1] w{ }^{*}\right] ; M i\{y[+m D i n t 2]=M I C\} D I P-d e l t a[M I 08287]$ & BDSC & $\begin{array}{l}\text { BDSC: } 51229 \\
\text { FBID: FBst0051229 }\end{array}$ \\
\hline $\begin{array}{l}y[1] w\left[^{*}\right] ; P\{w[+m C]=I o x(T r o j a n-G A L 4) \times 3\} 10 ; \operatorname{Dr}[1] / T M 3, \\
S b[1] \operatorname{Ser}[1]\end{array}$ & BDSC & $\begin{array}{l}\text { BDSC: } 60310 \\
\text { FBID: FBst0060310 }\end{array}$ \\
\hline$P\{y[+m D i n t 2]=C r e y\} 1 b, y[1] M\{$ vas-int.Dm\}ZH-2A w[*] & BDSC & $\begin{array}{l}\text { BDSC: } 60299 \\
\text { FBID: FBst0060299 }\end{array}$ \\
\hline$w\left[^{*}\right] ; P\{w[+m C]=U A S-C b b e t a \mid D T-A . l\} 18 / C y O$ (UAS-DTI) & BDSC & $\begin{array}{l}\text { BDSC: } 25039 \\
\text { FBID: FBst0025039 }\end{array}$ \\
\hline UAS-Dpr12 (in86FB) & This study & N/A \\
\hline UAS-DIP- $\delta$ (in attp40) & This study & N/A \\
\hline UAS-DIP-ס-2A-tdT & $\begin{array}{l}\text { A generous gift from } \\
\text { Larry Zipursky, UCLA }\end{array}$ & N/A \\
\hline$y[1] M\{w[+m C]=n o s-C a s 9 . P\} Z H-2 A w\left[^{*}\right]$ & BDSC & $\begin{array}{l}\text { BDSC: } 54591 \\
\text { FBID: FBst0054591 }\end{array}$ \\
\hline UAS-DIP-ס-RNAi (in attp40) & This study & N/A \\
\hline $\begin{array}{l}\left.y[1] w{ }^{\star}\right] \mathrm{P}\{\mathrm{y}[++\mathrm{t} 7.7] \mathrm{w}[+\mathrm{mC}]=10 X U A S-I V S- \\
\text { mCD8::RFP }\} \mathrm{su}(\mathrm{Hw}) \mathrm{attP} 8\end{array}$ & BDSC & $\begin{array}{l}\text { BDSC: } 32220 \\
\text { FBID: FBst0032220 }\end{array}$ \\
\hline \multicolumn{3}{|l|}{ Oligonucleotides } \\
\hline $\begin{array}{l}\text { gRNAs for Dpr12 CRISPR deletion: } \\
\text { CGCAGTTCCATCAGGTGCAGGGG } \\
\text { CCATTAGACATATCTTCCTGACC }\end{array}$ & This study & $\mathrm{N} / \mathrm{A}$ \\
\hline $\begin{array}{l}\text { Primers for dpr } 12^{\triangle 50-81} \text { check PCR: } \\
\text { F':GTTGCCGTAGCTGAAAGGATT } \\
\text { R':TAAACCGGGTATCGGAGTGTC }\end{array}$ & This study & N/A \\
\hline $\begin{array}{l}\text { RNAi for DIPס } \\
\text { (SS) GACGAUAAGAAACCUACAAUA } \\
\text { (AS) UUGUAGGUUUCUUAUCGUCAG }\end{array}$ & This study & N/A \\
\hline $\begin{array}{l}\text { Primers for QF2 cloning } \\
\text { F':TAAGCCAACTTTGAATCACAAGACGCATACCAAA } \\
\text { CGGTACATGCCACCCAAG } \\
\text { R':TGAATAATTTCTATTTGGCTTTAGTCGACGGTAT } \\
\text { CGATAATCACTGTTCGT }\end{array}$ & This study & N/A \\
\hline $\begin{array}{l}\text { Primers for hsp } 70 \text { cloning } \\
\text { F':AAGTGGTGATAAACGGCCGGCCGAGCGCCGGAG } \\
\text { TATAAATAGAG } \\
\text { R':AAGTGGTGATAAACGGCCGGCCGAGCGCCGGAG } \\
\text { TATAAATAGAG }\end{array}$ & This study & N/A \\
\hline \multicolumn{3}{|l|}{ Recombinant DNA } \\
\hline pCFD4 & (Port et al., 2014) & $\mathrm{N} / \mathrm{A}$ \\
\hline
\end{tabular}




\begin{tabular}{|c|c|c|}
\hline pVALIUM22 & $\begin{array}{l}\text { Harvard Medical } \\
\text { School }\end{array}$ & $\begin{array}{l}\text { PlasmID: } \\
\text { pVALIUM22 }\end{array}$ \\
\hline pDEST-UAS-IVS-Syn21-p10aw & $\begin{array}{l}\text { (Rabinovich et al., } \\
\text { 2016) }\end{array}$ & N/A \\
\hline pBPGUw & addgene & Plasmid \#17575 \\
\hline pBPGUw-QF2 & This paper & $\mathrm{N} / \mathrm{A}$ \\
\hline GMR71G10 entry vector & (Alyagor et al., 2018) & $\mathrm{N} / \mathrm{A}$ \\
\hline GMR71G10-QF2 ${ }^{\text {hsp70 }}$ & This paper & $\mathrm{N} / \mathrm{A}$ \\
\hline Plasmid: UAS-IVS-Syn21-Dpr12-p10 & This paper & $\mathrm{N} / \mathrm{A}$ \\
\hline Plasmid: UAS-IVS-Syn21-DIP-ס-p10 & This paper & N/A \\
\hline Plasmid: pCFD4-dpr12 (Dpr12 gRNA) & This paper & $\mathrm{N} / \mathrm{A}$ \\
\hline 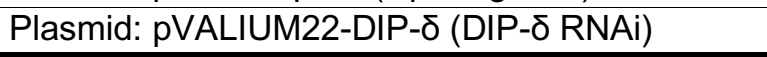 & This paper & $\mathrm{N} / \mathrm{A}$ \\
\hline \multicolumn{3}{|l|}{ Software and Algorithms } \\
\hline FIJI & Image $\mathrm{J}$ & $\begin{array}{l}\text { https://imagej.net/Fiji } \\
\text { /Downloads }\end{array}$ \\
\hline $\begin{array}{l}\text { VVD Viewer (a branch of FluoRender, Center for } \\
\text { Integrative Biomedical Computing (CIBC), Utah) }\end{array}$ & Takashi Kawase & $\begin{array}{l}\text { https://github.com/ta } \\
\text { kashi310/VVD_View } \\
\text { er/releases }\end{array}$ \\
\hline MATLAB R2016a software & MathWorks & N/A \\
\hline FlyMine & $\begin{array}{l}\text { Lyne et al } 2007 \text {, } \\
\text { Genome Biol }\end{array}$ & $\begin{array}{l}\text { http://www.flymine.or } \\
\mathrm{g} /\end{array}$ \\
\hline HISAT v.0.1.5 & (Kim et al., 2015) & $\begin{array}{l}\text { https://github.com/inf } \\
\text { philo/hisat }\end{array}$ \\
\hline DEseq2 & (Love et al., 2014) & N.A \\
\hline Gene-e v.3.0.215 & Broad Institute, Inc. & $\begin{array}{l}\text { https://software.broa } \\
\text { dinstitute.org/GENE- } \\
\text { E/ }\end{array}$ \\
\hline HOMER software & (Heinz et al., 2010) & \\
\hline DSIR & (Vert et al., 2006) & $\begin{array}{l}\text { http://biodev.extra.ce } \\
\text { a.fr/DSIR/DSIR.php }\end{array}$ \\
\hline FlyCRISPR & (Gratz et al., 2014) & $\begin{array}{l}\text { http://flycrispr.molbio } \\
\text {.wisc.edu/ }\end{array}$ \\
\hline \multicolumn{3}{|l|}{ Other } \\
\hline Zeiss LSM 800 confocal microscope & Zeiss & \\
\hline 40x 1.3 NA oil immersion lens & Zeiss & \\
\hline & & \\
\hline & & \\
\hline
\end{tabular}

\section{CONTACT FOR REAGENT AND RESOURCE SHARING}

Further information and requests for resources and reagents should be directed to and will be fulfilled by the Lead Contact, Oren Schuldiner (oren.schuldiner@weizmann.ac.il)

\section{EXPERIMENTAL MODEL}

Drosophila melanogaster flies were reared under standard laboratory conditions at $25^{\circ} \mathrm{C}$ on molasses containing food. Males and females were chosen at random. For developmental analysis white pupae were collected and incubated for the indicated number of hours. For adult analysis flies were dissected 3-5 days post eclosion. For a detailed list of the stocks and their source, see Key Resource Table. 


\section{GENOTYPES:}

hsFLP is y,w,hsFLP122; GFP is GFP; tdT is tdTom; mtdT-HA is mtdTomato-3xHA; 40A and $\mathrm{G} 13$ are FRTs on $2 \mathrm{~L}$ and $2 \mathrm{R}$ respectively; R71G10 is GMR71G10-Gal4; R18H09 is GMR18H09-Gal4; R58E02 is GMR58E02-Gal4; R10G03 is GMR10G03-Gal4; R48H011 is GMR48H011-Gal4; G80 is TubP-Gal80. Males and females were used interchangeably but only the female genotype is mentioned.

\section{Figure 1}

(D) y, w; R71G10, UAS-mCD8-GFP/+; R71G10, UAS-Dcr-2/+

(E) y, w; R71G10, UAS-mCD8-GFP/+; R71G10, UAS-Dcr-2/ TRiP.JF03210(dpr12RNAi)

(F) y, w; R71G10, UAS-mCD8-GFP/+; UAS-Cas9.C

(G) y, w; R71G10, UAS-mCD8-GFP/+; UAS-Cas9.C/dpr12-gRNA

(H-J) y, w; UAS-mCD8-GFP/+; R71G10/+

(K) y, w; UAS-mCD8-GFP/+; R71G10/UAS-Dpr12

(L-N) y, w; 40A, dpr12 ${ }^{\triangle 50-81}$, G13/40A, dpr12 $2^{\triangle 50-81}$, G13; R71G10, UAS-mCD8-GFP/+

(O) y, w; 40A, dpr12 $2^{\Delta 50-81}$, G13/40A, dpr12 ${ }^{\Delta 50-81}$, G13; R71G10, UAS-mCD8-GFP/UASDpr12

\section{Figure 2}

(A-C, G-I) hsFLP, UAS-mCD8-GFP; Gal80, FRT40A/FRT40A; R71G10, UAS-Dcr-2/+ (D-F, J-L) hsFLP, UAS-mCD8-GFP; Gal80, FRT40A/FRT40A; R71G10, UAS-Dcr-2/ TRiP.JF03210 (dpr12-RNAi)

(M) y, w; UAS-mCD8-GFP/+; R18H09/+

\section{Figure 3}

(A-C) y, w; Dpr12 ${ }^{\mathrm{GFSTF}} /+$

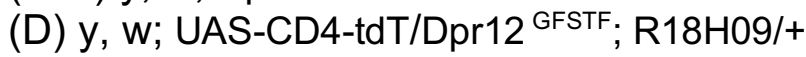

(E-G) y, w; DIP- $\delta^{\text {GFSTF } /+}$

(H) y, w; UAS-CD4-tdT/+; DIP- $\delta^{\text {GFSTF} / R 18 H 09 ~}$

\section{Figure 4}

(A-C) y, w; R71G10-QF2, QUAS-mtdT-HA/+; DIP- $\delta^{T 2 A-G a l 4 /+}$

(D) y, w; R71G10-QF2, QUAS-mtdT-HA/UAS-DIP- $\delta$; DIP- $\delta^{T 2 A-G a l 4 /+}$

(E-G) y, w; R71G10-QF2, QUAS-mtdT-HA/+; DIP- $\delta^{T 2 A-G a l 4} / D I P-\delta^{T 2 A-G a l 4}$

(H) y, w; R71G10-QF2, QUAS-mtdT-HA/UAS-DIP- $\delta ; D I P-\delta^{T 2 A-G a l 4} / D I P-\delta^{T 2 A-G a l 4}$

\section{Figure 5}

(A) UAS-mCD8-RFP; R53C03-p65.AD/+; DIP-ס GFSTF/R24E12-GAL4.DBD

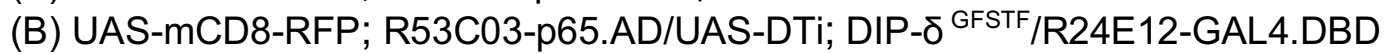

(C) y, w; UAS-CD4-tdT/+; DIP- $\delta$ GFSTF/R58E02

(D) y, w; UAS-CD4-tdT/UAS-DTi; DIP- $\delta$ GFSTF/R58E02

(E-G) hsFLP, UAS-mCD8-GFP; Gal80, FRT40A/FRT40A; DIP- $\delta^{T 2 A-G a l 4 /+}$

\section{Figure 6}

(A-D) y, w; Dpr12 ${ }^{\text {GFSTF }} /+; D I P-\delta^{T 2 A-G a l 4} / D I P-\delta^{T 2 A-G a l 4}$

(E-G) y, w; 40A, dpr12 ${ }^{\Delta 50-81}$, G13/40A, dpr12 $2^{\Delta 50-81}$, G13; DIP- $\delta^{\text {GFSTF } /+}$ 


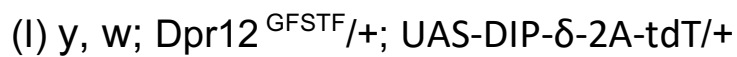

(J) y, w; Dpr12 ${ }^{\text {GFSTF } / R 30 G 08-p 65 . A D ; ~ R 48 B 03-G A L 4 . D B D / ~ U A S-D I P-\delta-2 A-t d T ~}$

Figure 7

(A) y, w; 40A, dpr12 $250-81$, G13/+; R10G03/UAS-mCD8-GFP

(B) y, w; 40A, dpr12 ${ }^{\triangle 50-81}, \mathrm{G} 13 / 40 \mathrm{~A}$, dpr12 ${ }^{\Delta 50-81}, \mathrm{G} 13$; R10G03/UAS-mCD8-GFP

(C) y, w; 40A, dpr12 ${ }^{450-81}, \mathrm{G} 13 /+;$ R48H011/UAS-mCD8-GFP

(D) y, w; 40A, dpr12 ${ }^{\Delta 50-81}, \mathrm{G} 13 / 40 \mathrm{~A}$, dpr12 ${ }^{\Delta 50-81}, \mathrm{G} 13$; R48H011/UAS-mCD8-GFP

(E) y, w; 40A, dpr12 $2^{450-81}, \mathrm{G} 13 /+$; R18H09/UAS-mCD8-GFP

(F) y, w; 40A, dpr12 ${ }^{\Delta 50-81}$, G13/40A, dpr12 $2^{\Delta 50-81}$, G13; R18H09/UAS-mCD8-GFP

(G) $w^{1118}$

(H) $w^{1118} ; 40 A$, dpr12 $12^{\Delta 50-81}, \mathrm{G} 13 / 40 \mathrm{~A}, \mathrm{dpr} 12^{\Delta 50-81}, \mathrm{G} 13$

\section{Figure $\mathbf{S 1}$}

(A)

y, w; R71G10, UAS-mCD8-GFP/+; R71G10, UAS-Dcr-2/ TRiP.JF03306(Dpr5-RNAi)

y, w; UAS-mCD8-GFP/+; R71G10/ TRiP.JF03172 (Dpr8-RNAi)

y, w; UAS-mCD8-GFP/+; R71G10/ TRiP.HMS00288 (Dpr9-RNAi)

y, w; R71G10, UAS-mCD8-GFP/+; R71G10, UAS-Dcr-2/ TRiP.JF02920 (Dpr10-RNAi)

y, w; R71G10, UAS-mCD8-GFP/+; R71G10, UAS-Dcr-2/ TRiP.JF03210(Dpr12-RNAi)

y, w; UAS-mCD8-GFP/+; R71G10/ TRiP.GL01238 (Dpr17-RNAi)

y, w; R71G10, UAS-mCD8-GFP/+; R71G10, UAS-Dcr-2/ TRiP.JF03283\}attP2 (Dpr18RNAi)

y, w; UAS-mCD8-GFP/+; R71G10/ TRiP.JF02923 (Dpr20-RNAi)

(D)

y, w; 40A, Dpr12 $2^{\Delta 50-81}$, G13/40A, Dpr12 ${ }^{\Delta 50-81}$, G13; R71G10, UAS-mCD8-GFP/+ y, w; 40A, Dpr12 $2^{\Delta 50-81}$, G13/40A, Dpr12 ${ }^{\Delta 50-81}$, G13; R71G10, UAS-mCD8-GFP/+ y, w; 40A, Dpr12 ${ }^{450-81}$, G13/40A, Dpr12 ${ }^{\Delta 50-81}$, G13; R71G10, UAS-mCD8-GFP/+ $y, w ;$ UAS-mCD8-GFP/+; R71G10/+

\section{Figure S2}

(A) hsFLP, UAS-mCD8-GFP; Gal80, FRT40A/FRT40A; R71G10, UAS-Dcr-2/+

(B) hsFLP, UAS-mCD8-GFP; Gal80, FRT40A/FRT40A; R71G10, UAS-Dcr-2/

TRiP.JF03210 (Dpr12-RNAi)

(D) y, w; UAS-mCD8-GFP/R30G08-p65.AD; R48B03-GAL4.DBD/ +

(E) y, w; UAS-mCD8-GFP/+; R18H09/ +

(F) y, w; UAS-mCD8-GFP/+; R48H011/+

Figure S3

(C-D) y, w; R71G10-QF2, QUAS-mtdT-HA/+; DIP- $\delta^{T 2 A-G a l 4 / D I P-\delta^{1-119}}$

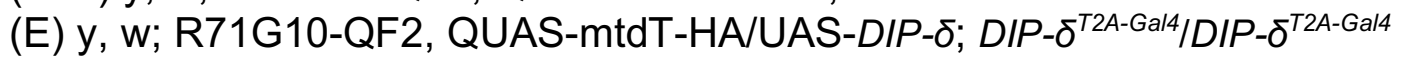

\section{Figure S4}

(A) y, w; UAS-DIP- $\delta$-RNAi/R71G10-QF2, QUAS-mtdT-HA; Repo-Gal4, UAS-mCD8-GFP/+ 
(B) y, w; UAS-DIP- $\delta$-RNAi/R71G10-QF2, QUAS-mtdT-HA; OK107-Gal4

(C) C155-Gal4; UAS-DIP-ס-RNAi/R71G10-QF2, QUAS-mtdT-HA

(D) y, w; UAS-DIP-ס-RNAi/R71G10-QF2, QUAS-mtdT-HA; DIP- $\delta^{T 2 A-G a l 4 /+}$

(E) C155-Gal4; UAS-Cas9.P2/+

(F) C155-Gal4; UAS-Cas9.P2/TKO.GS02451 (gRNA DIP-ס)

(G) y, w; R71G10, UAS-mCD8-GFP/ +; UAS-Cas9.C/+

(H) y, w; R71G10, UAS-mCD8-GFP/ TKO.GS02451(gRNA DIP-ס); UAS-Cas9.C/+

\section{Figure S5}

(A-C) y, w; QUAS-GFP, UAS-mtdT-HA/UAS-tdT; R58E02/R58E02-QF2

(D-F) y, w; R71G10-QF2, QUAS-mtdT-HA/+; DIP- $\delta^{T 2 A-G a / 4 /+}$

\section{Figure $\mathbf{S 6}$}

(A) y, w; 40A, dpr12 $2^{\Delta 50-81}$, G13/+; R10G03/UAS-mCD8-GFP

(B) y, w; 40A, dpr12 $2^{\Delta 50-81}, \mathrm{G} 13 / 40 \mathrm{~A}$, dpr12 $2^{\Delta 50-81}, \mathrm{G} 13$; R10G03/UAS-mCD8-GFP

(D) y, w; 40A, dpr12 ${ }^{450-81}, \mathrm{G} 13 /+;$ R48H011/UAS-mCD8-GFP

(E) y, w; 40A, dpr12 $2^{\Delta 50-81}$, G13/40A, dpr12 ${ }^{\triangle 50-81}$, G13; R48H011/UAS-mCD8-GFP

(G) y, w; 40A, dpr12 $250-81$, G13/+; R18H09/UAS-mCD8-GFP

(H) y, w; 40A, dpr12 ${ }^{\Delta 50-81}$, G13/40A, dpr12 ${ }^{\Delta 50-81}$, G13; R18H09/UAS-mCD8-GFP 


\section{METHOD DETAILS}

\section{RNA extraction}

The RNA extraction of WT and UNF-RNAi expressing MB $\mathrm{y}$ neurons was performed as described in Ref \#15. In brief, brains were dissected in a cold Ringer's solution and dissociated by incubation with collagenase/dispase mix at $29^{\circ} \mathrm{C}$ (Roche, 15 minutes for larval and pupal brains and 30 minutes for adult brains), washed in dissociation solution (Sigma-Aldrich), and mechanically dissociated into single cells. Cells were transferred via $35 \mu \mathrm{m}$ mesh (Falcon) to eliminate clusters and debris. $1000 \mathrm{y}$ neurons (DsRed ${ }^{+}$) were sorted using a $100 \mathrm{~mm}$ nozzle and low pressure in BD FACSAria Fusion (BD Bioscience) directly into $100 \mu \mathrm{l}$ Pico-Pure RNA isolation kit extraction buffer (Life Technologies) followed by RNA extraction. mRNA was captured using $12 \mathrm{ml}$ of Dynabeads oligo (Life Technologies), which were washed from unbound total RNA according to the protocol. mRNA was eluted from beads at $85^{\circ} \mathrm{C}$ with $10 \mathrm{ml}$ of $10 \mathrm{mM}$ Tris- $\mathrm{Cl}$ ( $\mathrm{pH}$ 7.5). mRNA was barcoded, converted into cDNA and linearly amplified by T7 in vitro transcription. The resulting RNA was fragmented and converted into an Illumina sequencing-ready library through ligation, RT, and PCR. Prior to sequencing, libraries were evaluated by Qubit fluorometer and TapeStation (Agilent).

\section{Analysis of RNA-Seq Data}

Samples were sequenced using Illumina NextSeq 500, at a sequencing depth of an average of 5 million reads. We aligned the reads to $D$. melanogaster reference genome (DM6, UCSC) using Hisat v0.1.5 with "-sensitive -local” parameters (Kim et al., 2015). Gene annotation were taken from FlyBase.org (Dmel R6.01/Fb_2014_04). Duplicate reads were filtered if they aligned to the same base and had identical unique molecular identifiers (UMI). Expression levels were counted using HOMER software (http://homer.salk.edu) (Heinz et al., 2010). For general analyses, we considered genes with reads over the noise threshold (20 reads). Significant expression in the $y$ neurons considered for genes with reads over a second noise threshold (50 reads) in at least two $y$ neuron. For normalization and statistics, we performed DEseq2 algorithm (Love et al., 2014) on our samples on $R$ platform, which took into account batch effects. All $p$ values presented for RNA-seq data are adjusted $p$ values. Gene enrichment analysis was done using FlyMine (http://www.flymine.org/).

\section{Generation of CRISPR mediated mutant}

For Dpr12 mutation two guide RNA were designed using the FlyCRISPR algorithm (http://flycrispr.molbio.wisc.edu/) and cloned into pCFD4 using Transfer-PCR (TPCR) (Unger et al., 2010, Melzer et al, under revision). The pCFD4-Dpr12 plasmid was injected into 86FB landing sites using ФC31 integration (BestGene). Injected flies were crossed with nanos-Cas9 flies (Bloomington stock \#54591). After two generations, single males were crossed with balancers and checked for deletion using specific primers. The Dpr12 ${ }^{\Delta 50-81}$ allele is a $32 \mathrm{bp}$ deletion in the 5' end of the transcript resulting in a premature stop after 37aa.

For tissue-specific CRISPR (tsCRISPR) Dpr12 and DIP-ס gRNA containing flies were crossed with UAS-Cas9.C or UAS-Cas9.P2 respectively, and the indicated Gal4s. 


\section{Generation of transgenes and transgenic flies}

To generate UAS-Dpr12 and UAS-DIP- $\delta$ transgenes, cDNA was cloned into the Gateway entry vector pDONR201. The Gateway entry vectors were then recombined into pDEST-UAS-IVS-Syn21-p10aw destination vector (Rabinovich et al., 2016) using LR recombinase (Invitrogen). UAS-Dpr12 and UAS-DIP- $\delta$ plasmids were injected into 86FB and attp40 landing sites, respectively, using $Ф C 31$ integration (BestGene).

For the generation of UAS-DIP- $\delta$ RNAi, 21 nucleotide sequence was selected using DSIR (http://biodev.extra.cea.fr/DSIR/DSIR.php). Off target results were eliminated by blast in NCBI. The RNAi hairpins were cloned into pVALIUM22 as described in https://fgr.hms.harvard.edu/cloning-and-sequencing. In brief, hairpins oligos, containing sense and anti-sense nucleotide with overhangs DNA fragment for Nhel and EcoRI were synthesized (Sigma). $10 \mu \mathrm{l}$ of sense and antisense strand oligos (10-20 $\mu \mathrm{M}$ each) were annealed into $80 \mu \mathrm{l}$ annealing buffer $(10 \mathrm{mM}$ Tris-HCL, pH7.5, $0.1 \mathrm{M} \mathrm{NaCl}, 1 \mathrm{mM}$ EDTA) by incubation at $95^{\circ} \mathrm{C}$ for $5 \mathrm{~min} .6 \mu$ of the annealed oligos were directly cloned in to pVALIUM22 vector which has been linearized by Nhel and EcoRI.

DIP- $\delta$ hairpins oligos (CAPS represent gene specific sequences): ctagcagtGACGATAAGAAACCTACAATAtagttatattcaagcataTTGTAGGTTTCTTATCGTC AGgcg aattcgcCTGACGATAAGAAACCTACAAtatgcttgaatataactaTATTGTAGGTTTCTTATCGT Cactg UAS-DIP- $\delta$ RNAi plasmid was injected into attp40 landing sites, using ФC31 integration (BestGene).

\section{Generation of DIP- $\delta^{T 2 A-G a l 4}$}

$D I P-\delta^{T 2 A-G a / 4}$ was generated as described in (Diao et al., 2015). In brief, flies carrying the MiMICM108287 insertion were crossed with the flies bearing the triplet donor cassettes (Trojan Gal4 cassettes of the three reading frames). Males from this progeny carrying both components were crossed to females carrying germline transgenic sources of Cre and $\Phi C 31$. Adult progeny with all relevant components were crossed to UAS-GFP balanced on the $3^{\text {rd }}$ chromosome. Single males from this final cross were screened by fluorescence microscopy for Gal4 expression.

\section{Generation of QF2 driver}

To generate the R71G10-QF2 driver, the QF2 sequence was amplified from pattBDSCP_prom-QF7-hsp70_term (a gift from Chris potter) using the QF-F and QF-R primers, and cloned into pBPGUw plasmid, using the Gibson assembly kit (NEB) to create pBPGUw-QF2. Then the GMR71G10 entry vector (Alyagor et al., 2018)was recombined into pBPGUw-QF2 using LR recombinase (Invitrogen). Finally, the DSCP promotor region within the GMR71G10-QF2 was replaced with hsp70 promotor by RF cloning using the hspF and hspR primers. The GMR71G10-QF2 ${ }^{\text {hsp70 }}$ plasmid was injected into attp40 landing sites, using $\Phi C 31$ integration (BestGene). QF-F TAAGCCAACTTTGAATCACAAGACGCATACCAAACGGTACATGCCACCCAAG QF-R

TGAATAATTTTCTATTTGGCTTTAGTCGACGGTATCGATAATCACTGTTCGT $\mathrm{HspF}$ 


\section{AAGTGGTGATAAACGGCCGGCCGAGCGCCGGAGTATAAATAGAG HspR \\ AAGTGGTGATAAACGGCCGGCCGAGCGCCGGAGTATAAATAGAG}

\section{Generation of MARCM clones}

Due to Dpr12 centromeric chromosomal location we could not generate dpr12 mutant clones and instead expressed dpr12-RNAi within y neuron clones. MB y neuron MARCM clones were generated as described in (Lee and Luo, 1999). In brief, flies were heat shocked (hs) for $40-60 \mathrm{~min}$ at $37^{\circ} \mathrm{C}$ at $24 \mathrm{hr}$ after egg laying and examined the indicated developmental time points.

To discover the mitotic window which results in adult PAM neurons, we used the MARCM technique to generate DIP- $\delta^{T 2 A-G a l 4}$ clones by hs at different developmental times. Only hs at $0-24 \mathrm{hr}$ after egg laying resulted in clones containing PAM neurons, and therefore this hs regime was used in this study. Importantly, here mitotic recombination was performed using FRT40A and used to eliminate Gal80 expression but $D I P-\delta$ remained heterozygous as it is on another chromosome.

\section{Immunostaining and imaging}

Brains were dissected in ringer solution, fixed using 4\% paraformaldehyde (PFA) for 20 minutes at room temperature (RT) and washed with PB with $0.3 \%$ Triton-X (PBT, 3 immediate washes followed by $3 \times 20$ minute washes). Non-specific staining was blocked using $5 \%$ heat inactivated goat serum in PBT and then samples were subjected to primary antibodies (over-night, $4^{\circ} \mathrm{C}$ ) and secondary antibodies ( 2 hours at RT) with PBT washes ( 3 quick washed followed by $3 \times 20$ minute washes). The brains were mounted on SlowFade (Invitrogen) and imaged using Zeiss LSM800 confocal microscope. Images were processed with ImageJ $1.51(\mathrm{NIH})$. Individual neurons were traced manually through all focal planes using the Edge Detection Settings of the Analyze Paint Brush VVD selection tool (Takashi Kawase). Thresholding was individually adapted for each focal plane and neuronal structures and refined through the Analyze Erase tool whenever necessary.

For Brp staining brains were blocked in $2 \%$ bovine serum albumine (BSA) in PBT for 2 $\mathrm{hr}$ at RT, incubated with mouse anti-brp antibody for two days at $4^{\circ} \mathrm{C}$ then incubated with secondary antibody for an over-night at $4^{\circ} \mathrm{C}$. Next, brains were transferred to a poly-L-lysine (Sigma Aldrich. \# P1524-25MG) pre-treated cover glasses (22x22x1; Fisher Scientific. \# 12-542B), fixed, dehydrated in ascending alcohol series $(30 \%, 50 \%$, $75 \%, 95 \%$ and $3 \times 100 \%, 10$ min each), incubated in Xylene $2 \times 10$ min and embedded in DPX (Electron Microscopy Sciences; \# 180627-05) and incubated for at least 4 days. Confocal laser scanning microscopy was done using an Olympus microscope equipped with a Plan-Apochromat 20x objective. Taken images were analyzed using VVD Viewer (Takashi Kawase).

\section{Quantification and statistical analysis}

In all cases, statistical significance was calculated as follows: *** represent a P-value lower than $0.001 ;{ }^{* *}$ represent a P-value lower than 0.01 and * represents a P-value lower than 0.05 . Specific $p$-values and sample sizes are indicated in the relevant figure legends. 
For quantification of regrowth (Figure 1, and 4), confocal Z-stacks were given to an independent lab member who blindly ranked the severity of the regrowth defects. For statistical analysis Kruskal-Wallis test was performed followed by a Wilcoxon-MannWhitney test post-hoc test. 
Bornstein et al. SFigure 1
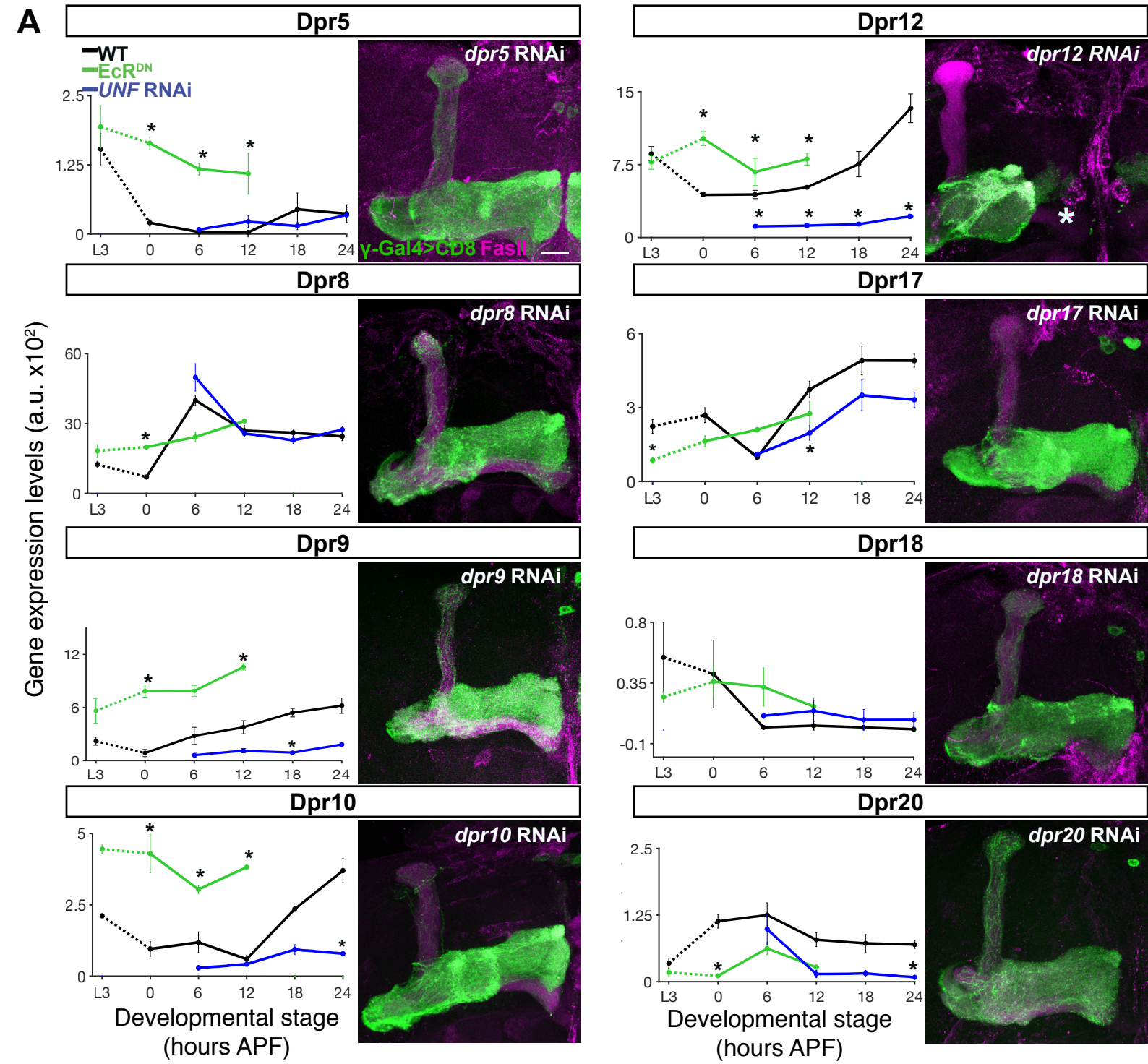

B

dpr12 $2^{\Delta 50-81}$

CRISPR 32bp deletion

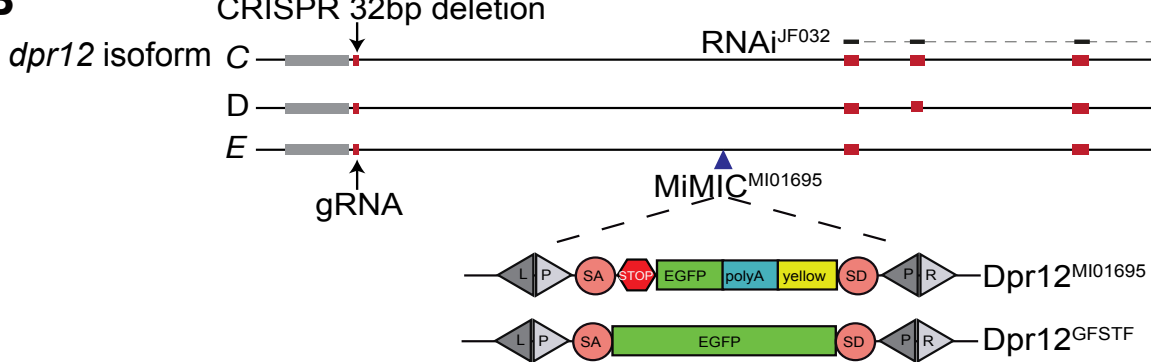

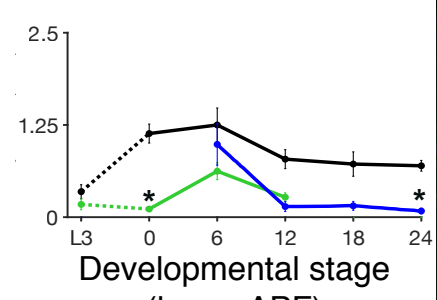

(hours APF)

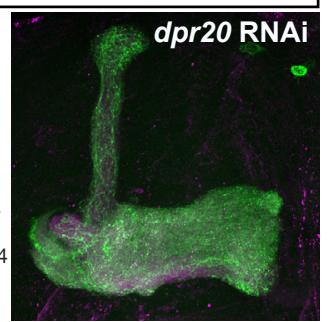

C Dpr12 N $\mathrm{N}^{\mathrm{SP}} \mathrm{lg}-\mathrm{lg} \stackrel{\text { TM/GPI }}{\mathrm{T}} \mathrm{C}^{\prime}$

Dpr12 $2^{\triangle 50-81} \mathrm{~N}^{\prime} \cdot \mathrm{HC}^{\prime}$

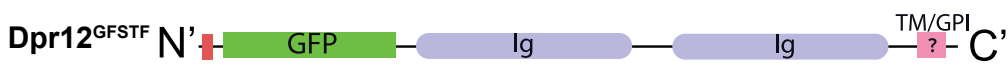

D

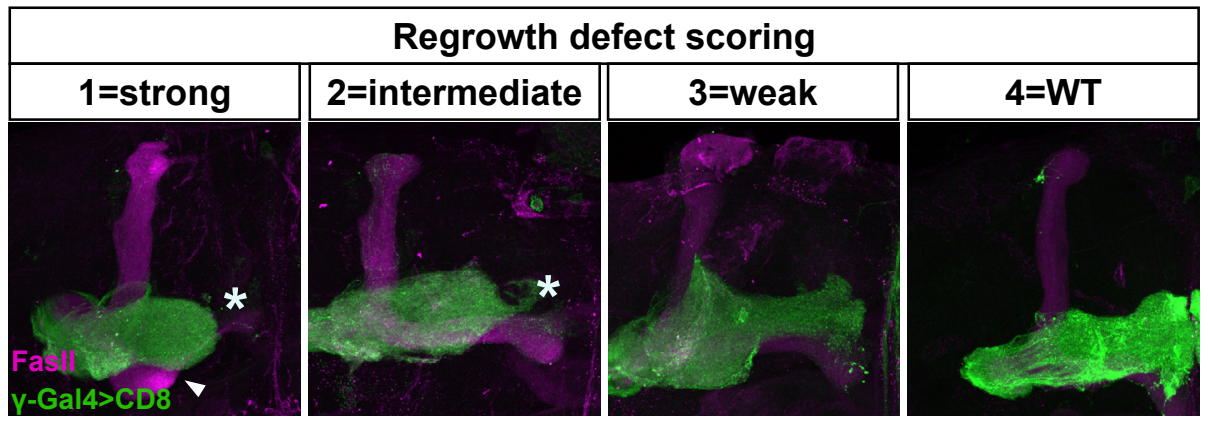




\section{Supplemental Figures and Legends}

Figure S1. Knock down of dpr12 results in regrowth phenotype and description of Dpr12 alleles used in the study, related to Figure 1.

(A) Left: Graphs depicting the normalized RNA expression levels of selected Dprs in WT $\mathrm{Y}$-KCs (black), and in $\mathrm{Y}-\mathrm{KC}$ s expressing EcR ${ }^{\mathrm{DN}}$ (green) or UNF-RNAi (blue). * $\mathrm{p}<0.05$; Error bars indicate SEM; units on the y axis are arbitrary. Right: Confocal z-projections of adult $\mathrm{Y}$-KCs expressing RNAi transgenes as indicated labeled with membrane bound GFP (mCD8-GFP; CD8) driven by the y specific Gal4 driver GMR71G10-Gal4 ( $y-G a l 4)$.

(B) A schematic representation of the Dpr12 locus showing introns (black line), coding and non-coding exons (red and gray, respectively). The location of Dpr12 gRNA (arrow), dpr12 ${ }^{\triangle 50-81}$ mutation (arrow), dpr12 RNAijF03210 (black lines connected with

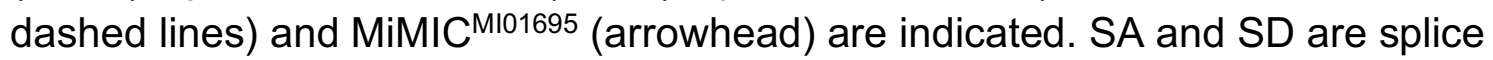
acceptor and donor sites, respectively. Recombination mediated cassette exchange was used to transform Dpr12 ${ }^{\mathrm{M} 101695}$ into Dpr12 ${ }^{\mathrm{GFSTF}}$.

(C) A schematic representation of Dpr12 protein variants. Signal peptide (SP), Immunoglobulin (lg), transmembrane (TM) and GPI anchor (GPI).

(D) Ranking of regrowth: Confocal z-projections of adult $\mathrm{Y}-\mathrm{KC}$ s labeled with membrane bound GFP (mCD8-GFP; CD8) driven by the y specific Gal4 driver GMR71G10-Gal4 ( $\gamma$ Gal4). Representative images of the regrowth defect severity (1=strong, 2=intermediate, $3=$ weak, $4=\mathrm{WT}$ ) described in Figure 10. Arrowhead demarcates short $\beta$ lobe, which appear in approximately $40 \%$ of $d p r 12$ homozygous mutant brains, but not in $d p r 12-$ RNAi expressing brains.

Asterisk demarcates the distal tip of the $y$ lobe. Green is CD8-GFP; magenta is Fasll staining; Scale bar is $20 \mu \mathrm{m}$. 

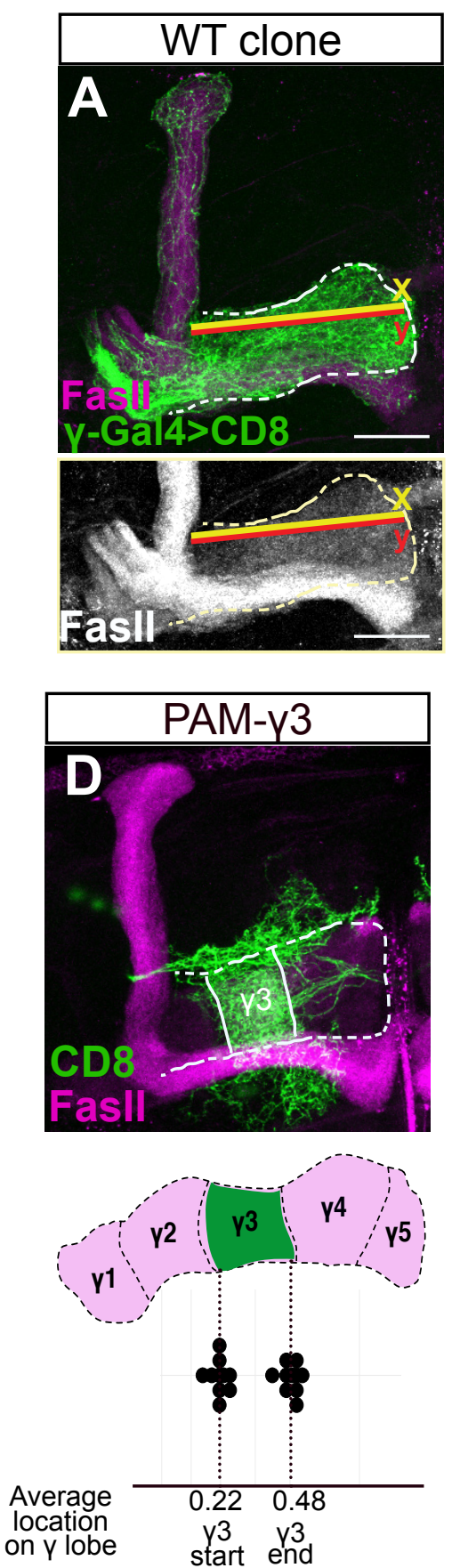
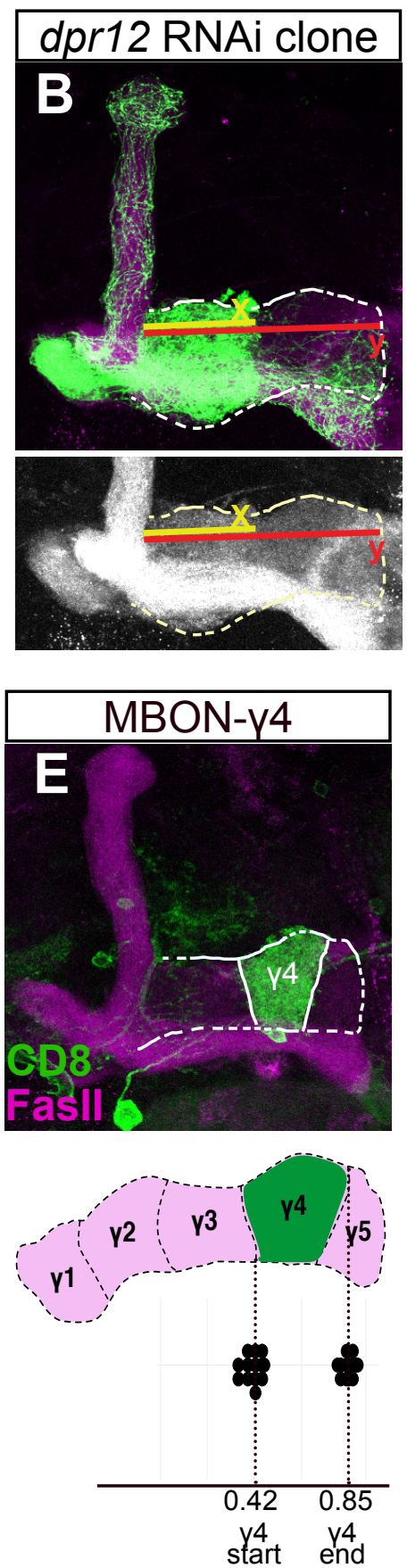

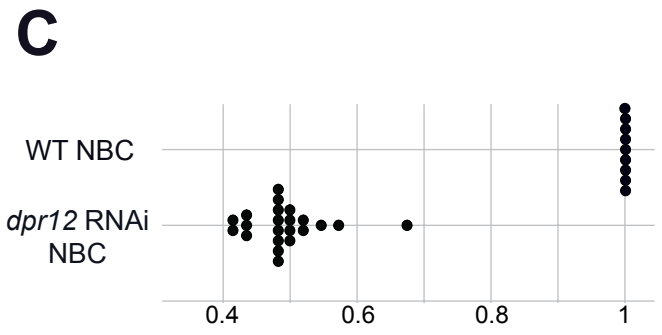

Relative location on y lobe (x/y ratio)
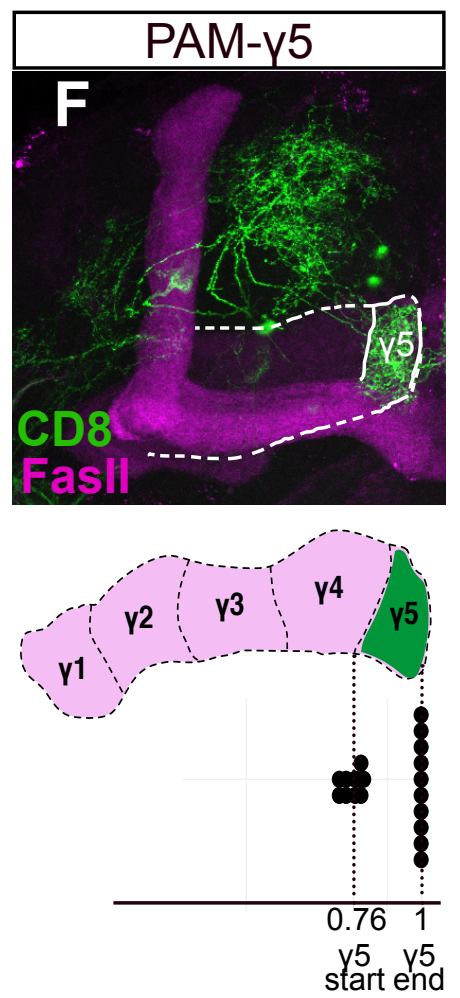
Figure S2. Measurements of $\mathrm{Y}$-axon outgrowth, related to Figure 2.

(A-B) Confocal z-projections of WT (A) and dpr12 RNAi (B) MARCM neuroblast clones (NBC) labeled with membrane bound GFP (mCD8-GFP; CD8) driven by the y specific Gal4 driver GMR71G10-Gal4 (

$y$ (red) represents the length of the entire $y$-lobe as indicated; $x$ (yellow) represents the extent of clonal $y$-axon outgrowth.

(C) Measurements of the $x / y$ ration as depicted in A-B. While WT NBC always extend up to the end of the lobe, dpr12 RNAi NBC stop at about midway (x/y ratio of $0.48 \pm$ 0.05).

(D-F) Top: Confocal z-projections of PAM-ү3 (D, MB441B), MBON-ү4 (E, R18H09), and PAM-y5 ( $F, R 48 H 11)$ Gal4s driving the expression of mCD8-GFP (CD8). Bottom: start and end of the indicated zone is superimposed on a schematic representation of the adult $y$ lobe compartments. (D) $\gamma 3$ zone begins at $x / y$ ratio of $0.22 \pm 0.03$ and ends at $0.48 \pm 0.03$. (E) $y 4$ zone begins at $x / y$ ratio of $0.42 \pm 0.04$ and ends at $0.85 \pm 0.03$. (F) y5 zone begins at $x / y$ ratio of $0.76 \pm 0.03$ and ends at a mean ratio of 1 .

Green is CD8-GFP; magenta and white represent Fasll; Scale bar is $20 \mu \mathrm{m}$. 


\section{Bornstein et al. SFigure 3}

A

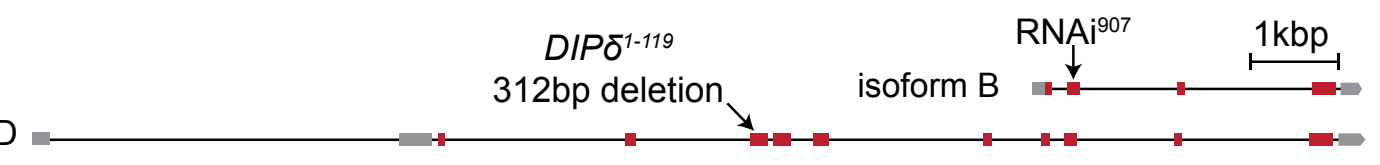

$D I P \delta$ isoform D

E

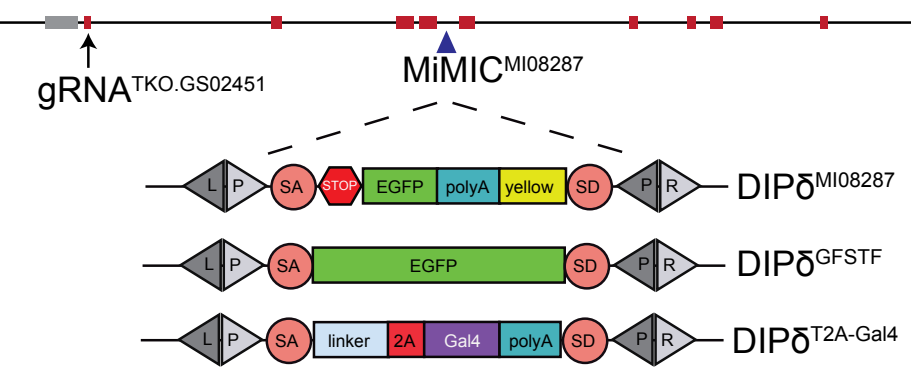

B
DIPס
$\mathrm{N}^{\prime}-\mathrm{S}$
$\lg$
DIPSGFSTF
$\mathrm{N}+\mathrm{SP}$
$\lg$
GFP
DIP $\delta^{\mathrm{T} 2 \mathrm{~A}-\mathrm{Gal} 4}$
$\mathrm{N}+\mathrm{S}$
$19-9$
DIP ${ }^{1-119}$
$\mathrm{N}^{\mathrm{S}-\mathrm{S}}$
Ig -TC'

Ig $\quad$ GPI $C^{\prime}$

Ig $\quad$ GPI ? $^{\prime}$

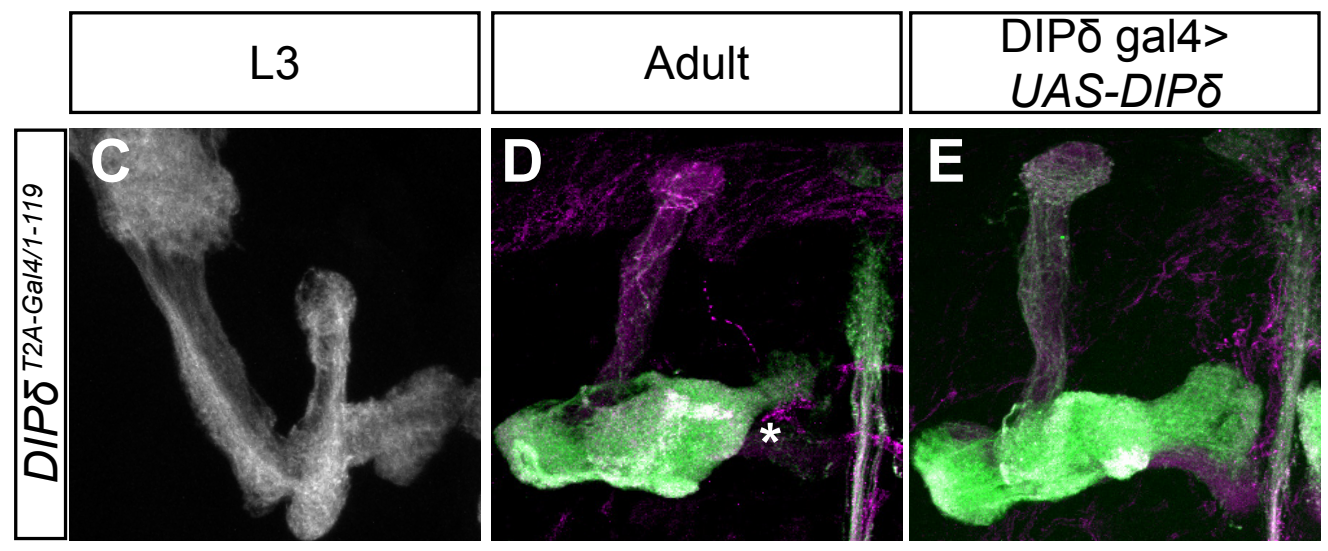


Figure S3. Description of DIP- $\delta$ alleles used in the study and additional DIP- $\delta$ loss of function phenotypes, related to Figure 4.

(A) A schematic representation of the DIP- $\delta$ locus showing introns (black line), coding and non-coding exons (red and gray, respectively). The location of DIP- $\delta$ gRNA, DIP- $\delta^{1-}$ 119 mutation, DIP- $\delta$ RNAi and MiMIC ${ }^{\text {MI08287 }}$ are indicated. SA and SD are splice acceptor and donor sites, respectively. Recombination mediated cassette exchange was used to transform DIP- $\delta^{\text {MI08287 }}$ into DIP- $\delta^{\text {GFSTF }}$ and DIP- $\delta^{\text {T2A-Gal4 }}$.

(B) A schematic description of DIP- $\delta$ protein variants. Signal peptide (SP), Immunoglobulin (Ig), and GPI anchor (GPI).

(C-E) Confocal z-projections of DIP- $\delta$ transheterozygotes (DIP- $\delta^{\mathrm{T} 2 \mathrm{~A}-\mathrm{Gal} 4 / 1-119}$ ) at L3 (C; $n=20 / 20)$ and adult ( $D ; n=12 / 12, E ; n=22 / 23)$, in which in which $y$ neurons were marked by expressing membrane bound tandem tomato (mtdT-HA) driven by the $Y$ specific QF2 driver GMR71G10-QF2 ( $\gamma-Q F 2)$. In DIP- $\delta$ transheterozygotes, as in homozygous mutants (see Figure 4), y neurons do not extend into the distal end of the lobe. Expression of DIP- $\delta$ in DIP- $\delta^{+}$cells $(E)$, rescues the growth defect present in DIP- $\delta^{T 2 A-}$ Gal4/1-119 brains.

Green and white represent mtdT-HA; magenta represents Fasll staining; Scale bar is $20 \mu \mathrm{m}$. 
Bornstein et al. SFigure 4
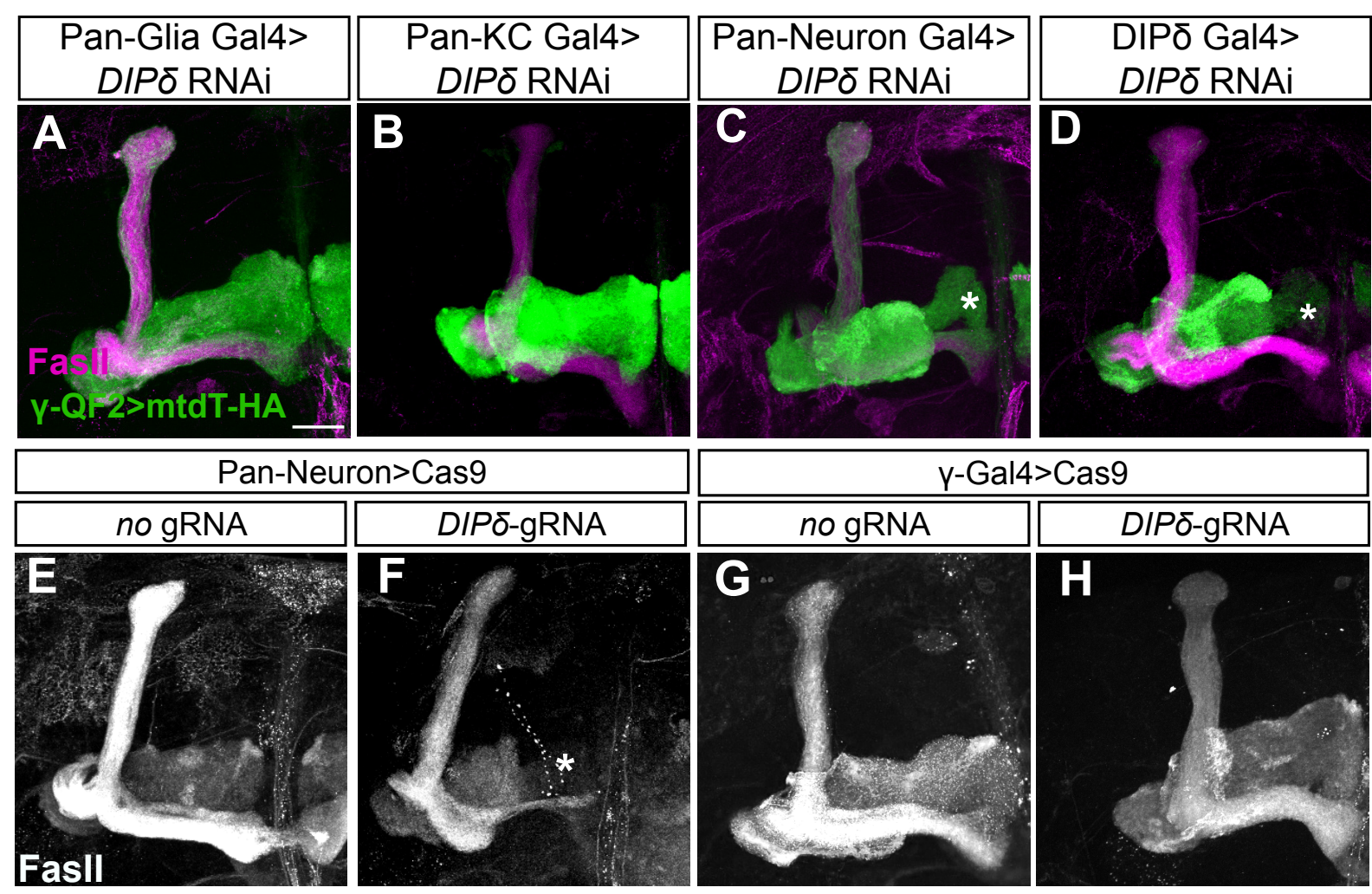

DIPס-gRNA
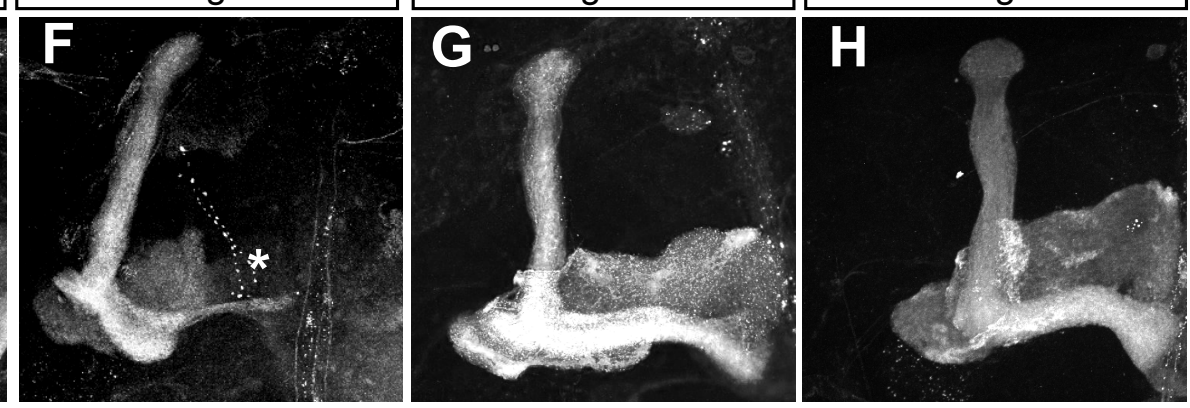
Figure S4. DIP-ס is non-cell-autonomously required for $\mathrm{y}$-axon regrowth, related to Figure 4.

(A-D) Confocal z-projections of brains expressing DIP- $\delta$-RNAi driven by the indicated Gal4. $Y-K C$ s are labeled by membrane bound tandem tomato (mtdT-HA) driven by the $Y$ specific QF2 driver GMR71G10-QF2 ( $\gamma-Q F 2)$. Expression of DIP- $\delta$-RNAi in all glia (Repo-Gal4, L; n=28/28) or all KCs (OK107-Gal4, M; n=12/12) did not affect $y$ neuron regrowth. In contrast, expression of DIP- $\delta$-RNAi in all postmitotic neurons (C155-Gal4, $\mathrm{N} ; \mathrm{n}=9 / 12)$ or DIP- $\delta$ expressing neurons $\left(D I P-\delta^{T 2 A-G a / 4}, O ; n=21 / 22\right)$ induced a defect in $\mathrm{Y} 4 / 5$ innervation by $\mathrm{Y}$-axons.

$(E-H)$ Confocal z-projections of brains expressing UAS-Cas9 alone $(E, G)$ or together with DIP- $\delta$-gRNA $(\mathrm{F}, \mathrm{H})$. DIP- $\delta$ knockout by tsCRISPR in all postmitotic neurons (F; $\mathrm{n}=22 / 24$ ) resulted in a defect in $\gamma 4 / 5$ innervation by $\gamma$-axons, while $D I P-\delta$ knockout by tsCRISPR in $\mathrm{y}$-KCs $(\mathrm{H} ; \mathrm{n}=28 / 28)$ did not affect $\mathrm{y}$-axon regrowth. Expression of Cas9 alone $(E, n=10 / 10 ; G, n=14 / 14)$ did not affect $y$-axon regrowth.

Green is mtdT-HA; magenta and white represent Fasll staining; Scale bar is $20 \mu \mathrm{m}$. 
Bornstein et al. SFigure 5
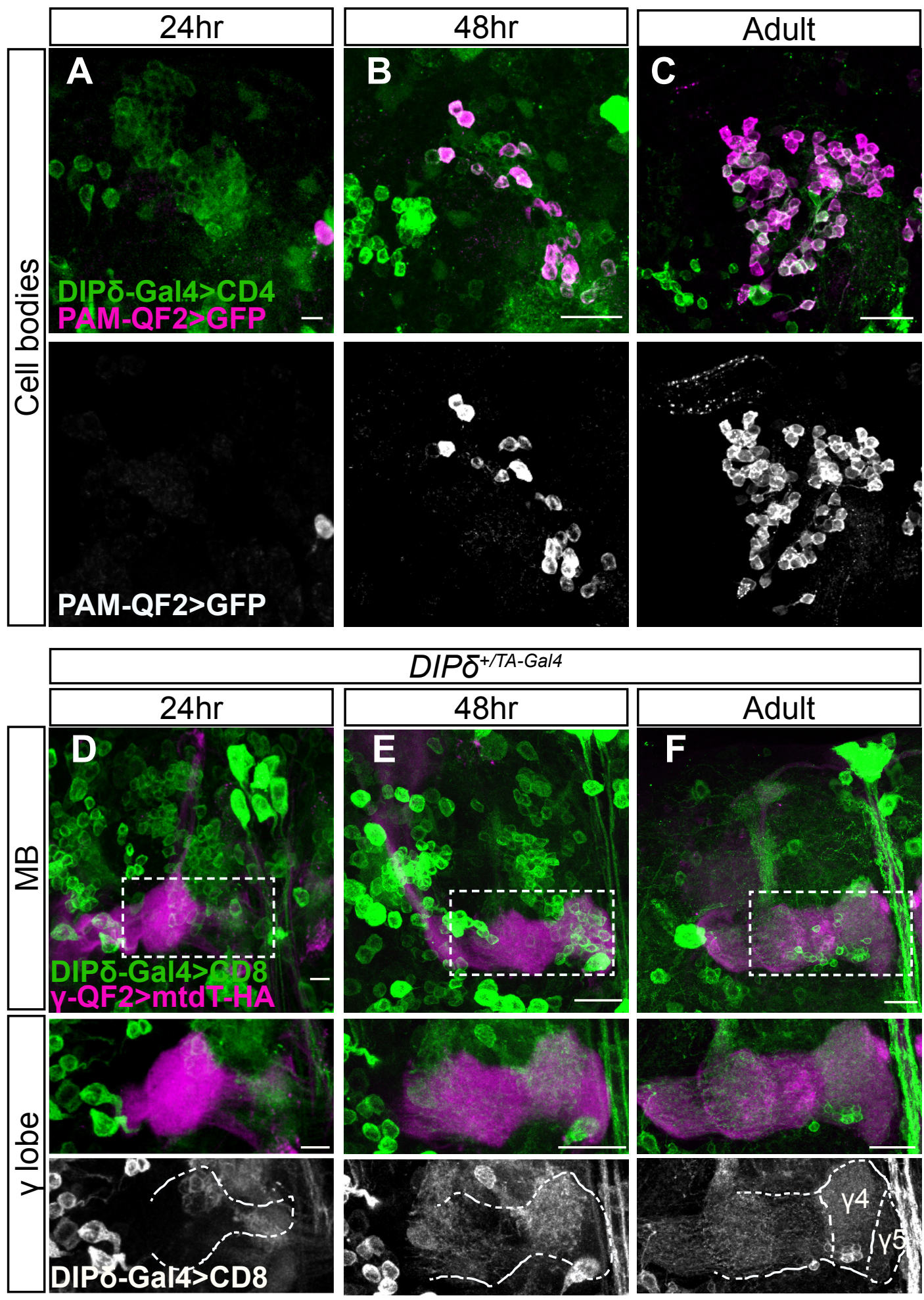
Figure S5. Characterization of PAM and DIP-ס Gal4s, related to Figure 5.

(A-C) Confocal z-projections of the cell body region of the PAM cluster demonstrating the expression of membrane bound tandem tomato (CD4-tdT; CD4) driven by DIP- $\delta^{T 2 A-}$ Gal4 (DIP-ס-Gal4) in addition to GFP driven by the PAM specific QF2 driver GMR58E02QF2 (PAM-QF2). The PAM-QF2 driver is not expressed at 24hr APF $(A, n=12)$, starts to be expressed at $48 \mathrm{hr}$ APF $(B, n=10)$ and fully expressed and localized with DIP- $\delta-G a l 4$ in adult $(C, n=14)$.

(D-F) Confocal z-projections of heterozygous brains (DIP- $\left.\delta^{+/ T 2 A-G a l 4}\right)$ in which DIP- $\delta$ positive neurons were labeled by membrane bound GFP (mCD8-GFP; CD8) driven by

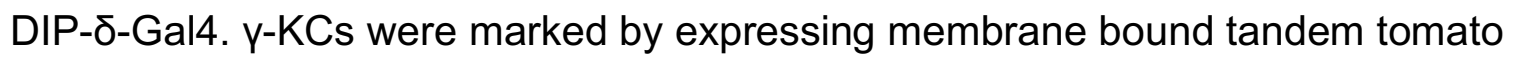
(mtdT-HA) driven by the $y$ specific QF2 driver GMR71G10-QF2 (Y-QF2). Bottom: high magnification images, as demarcated by dashed box in top panels.

Dashed outline demarcates $\gamma$-lobe as depicted by Fasll staining. $\gamma 4 / 5$ zones are indicated in adult. $D, n=20 ; E, n=16 ; F, n=26$.

Scale bar is $20 \mu \mathrm{m}$. 
Bornstein et al. SFigure 6
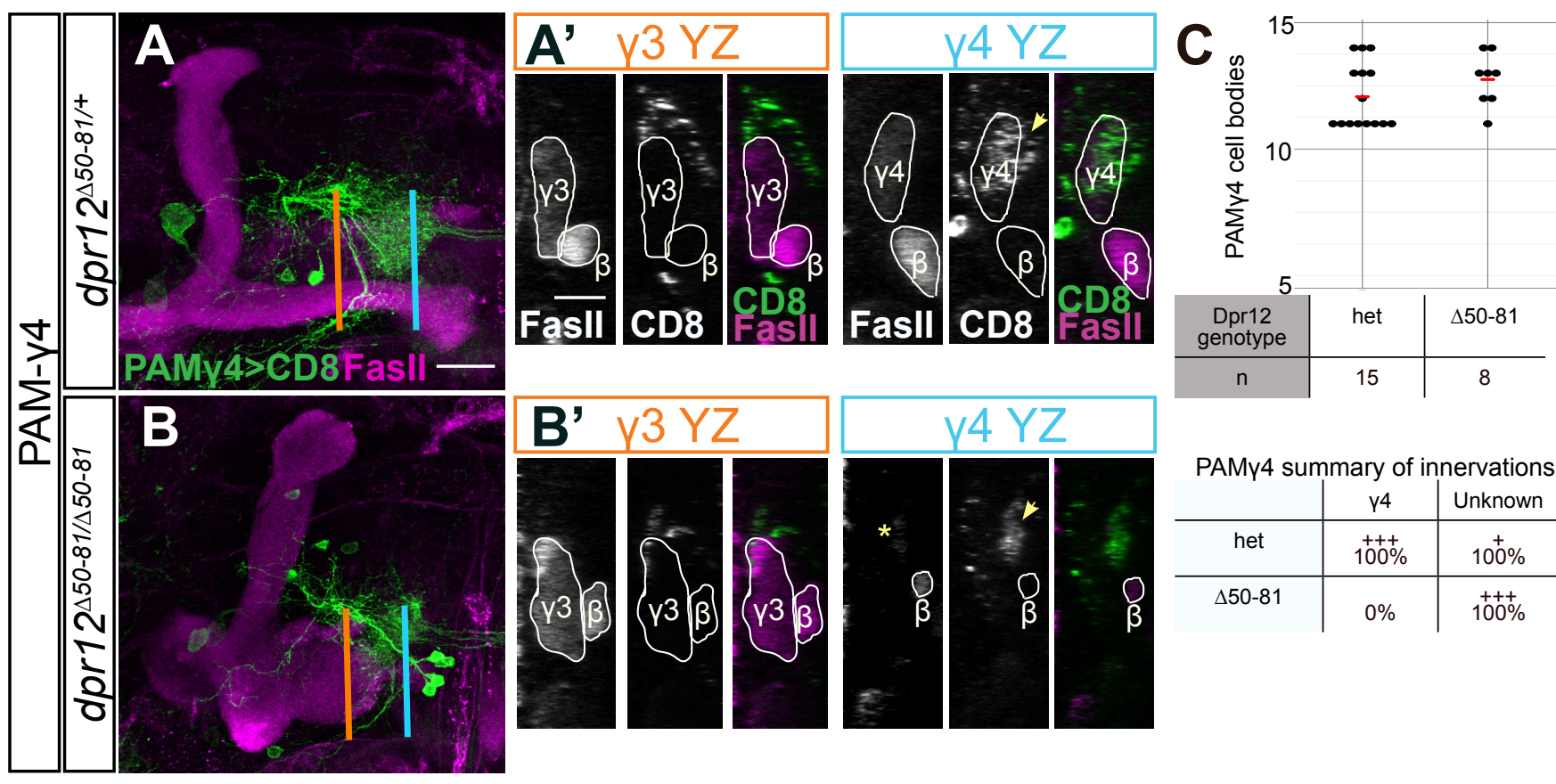

PAMy4 summary of innervations
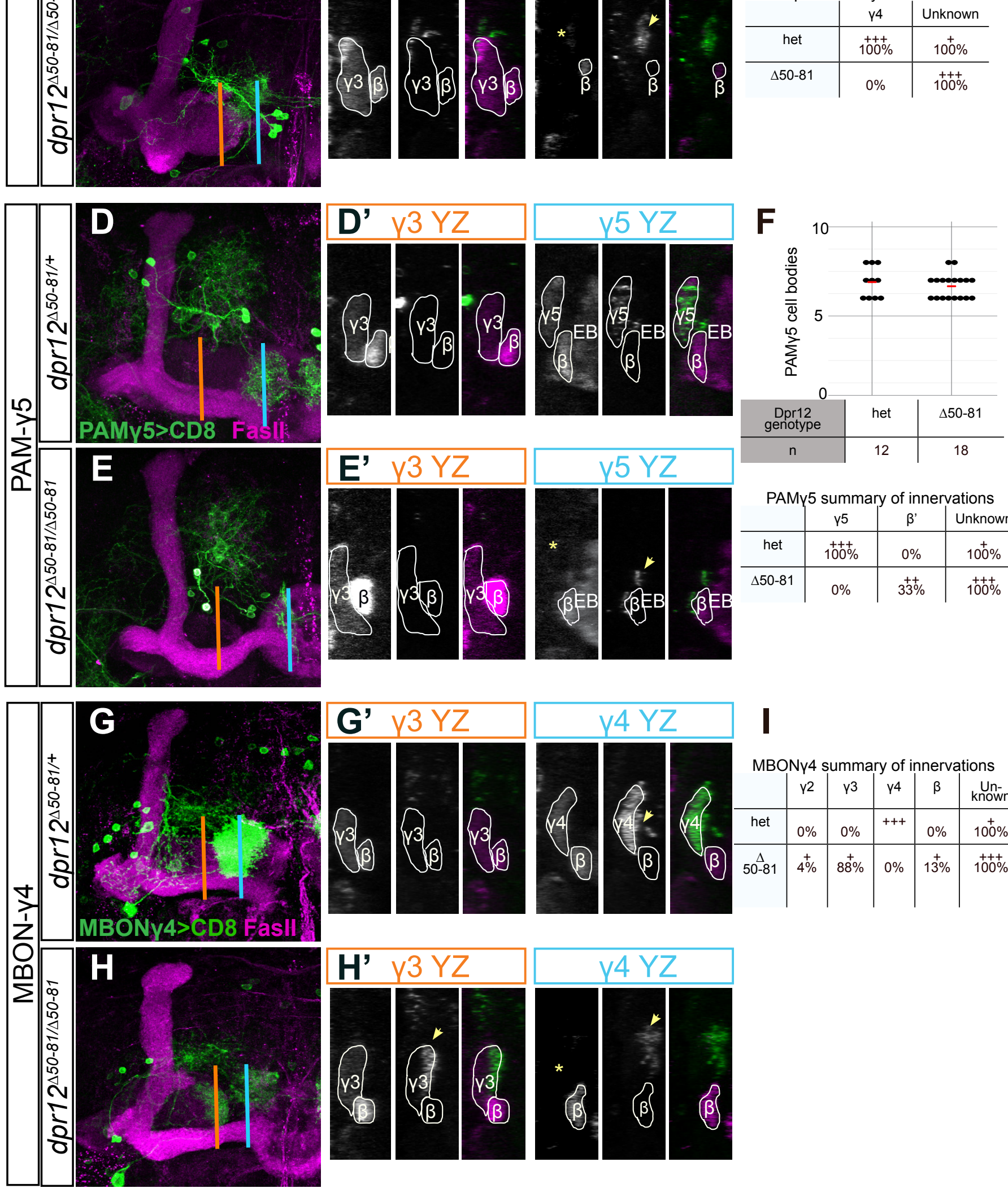

PAMy5 summary of innervations

\begin{tabular}{c|c|c|c} 
& $\gamma 5$ & $\beta^{\prime}$ & Unknown \\
\hline het & $\begin{array}{c}+++ \\
100 \%\end{array}$ & $0 \%$ & $100 \%$ \\
\hline$\Delta 50-81$ & $0 \%$ & $33 \%$ & $100 \%$ \\
& $3++++$ \\
\end{tabular}
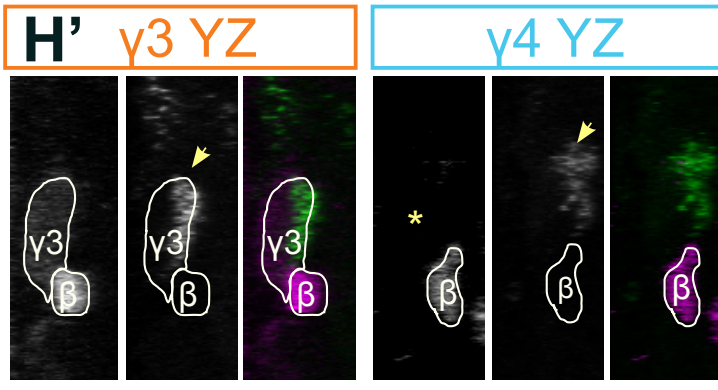

MBONy4 summary of innervations

\begin{tabular}{c|c|c|c|c|c} 
& $\mathrm{Y} 2$ & $\mathrm{\gamma} 3$ & $\mathrm{\gamma}$ & $\beta$ & $\begin{array}{c}\text { Un- } \\
\text { known }\end{array}$ \\
\hline het & $0 \%$ & $0 \%$ & +++ & $0 \%$ & $100 \%$ \\
\hline $\begin{array}{c}\Delta \\
50-81\end{array}$ & $\stackrel{+}{4} \%$ & $\stackrel{+}{8} \%$ & $0 \%$ & $13 \%$ & $\stackrel{++++}{100 \%}$ \\
\end{tabular}


Figure S6. Phenotypic analysis of PAM and MBON innervation of the MB $Y$-lobe in Dpr12 mutant brains, related to Figure 7.

(A-B, D-E, G-H) Left: Confocal z-projections of $d p r 12$ heterozygous or homozygous mutant brains (also shown in Figure 6) expressing membrane bound GFP (CD8) driven by: (A-B) R10G03-Gal4 is used to label PAM neurons innervating the $\mathrm{Y} 4$ compartment (PAM-ү4); (D-E) R48H11-Gal4 is used to label PAM neurons innervating the $\mathrm{y} 5$ compartment (PAM- $\mathrm{Y} 5)$; (G-H) R18H09-Gal4 is used to label MBON innervating the $\mathrm{Y} 4$ compartment (MBON-ү4). Right: $\mathrm{YZ}$ projections along the indicated lines in $\mathrm{\gamma} 3$ (orange) and $\mathrm{\gamma} 4$ or $\mathrm{y} 5$ (blue) compartments.

(C,F,I) Top: Cell body numbers of the indicated neurons in dpr12 heterozygous and homozygous brains; Bottom: Summary of innervation destinations. Unknown means stereotypic projections to unidentifiable domains.

Green is CD8-GFP, magenta is Fasll, grayscale single channels are shown as indicated. Asterisks mark missing innervation and arrows mark innervations outside the $y$ lobe. Scale bar is $20 \mu \mathrm{m}$ in A-B, D-E, G-H and $10 \mu \mathrm{m}$ in A'-B', D'-E', G'-H'.

\section{Movie legends and Table descriptions}

\section{Movie S1: Tracing of control y single cell clones}

Confocal reconstructions control MARCM y single cell clones. Stacks were taken from Figure 2I, and used to trace single axons in Figure 2l' which were included in the quantification in Figure 2N.

\section{Movie S2: Tracing of dpr12-RNAi y single cell clones}

Confocal reconstructions MARCM y single cell clones expressing dpr12-RNAi. Stacks were taken from Figure 2L, and used to trace single axons in Figure 2L' which were included in the quantification in Figure $2 \mathrm{~N}$.

\section{Movie S3: Structure of $\mathrm{Y}$-lobe compartments in WT brain}

Confocal reconstruction of a control brain $\left(w^{*}\right)$ stained with anti-Brp (the same brain that is presented in Figure 7G). The adult $y$-lobe compartments ( $\gamma 1-\gamma 5)$ are marked with yellow, g green, cyan, blue and light blue. The adult $\alpha \beta$ and $\alpha$ ' $\beta$ ' neurons are marked with magenta and red respectively. Scale bar is $20 \mu \mathrm{m}$.

Movie S4: Structure of $y$-lobe compartments in dpr12 mutant brain Confocal reconstruction of $d p r 12$ homozygous mutant brain stained with anti-Brp (as presented at Figure $7 \mathrm{H})$. The adult $\mathrm{\gamma}$-lobe compartments $(\mathrm{\gamma} 1-\mathrm{\gamma} 4)$ are marked with 
yellow, green, cyan, and blue. Note that the $\gamma 5$ and most of the $\gamma 4$ compartments are missing. The adult $\alpha \beta$ and $\alpha^{\prime} \beta$ ' neurons are marked with magenta and red respectively. Scale bar is $20 \mu \mathrm{m}$.

Table S1: Normalized RNAseq data

Normalized and averaged RNAseq profiling of WT MB y neurons during development (taken from ref 14), compared to expression profiles of MB y neurons expressing UNFRNAi.

Table S2: Expression analysis of the Dpr/DIP families

Normalized and averaged RNAseq values of Dpr and DIP transcripts in WT MB Y neurons during development (taken from ref 14), compared to expression profiles of MB Y neurons expressing UNF-RNAi. 


\section{Supplemental References:}

Alyagor, I., Berkun, V., Keren-Shaul, H., Marmor-Kollet, N., David, E., Mayseless, O., Issman-Zecharya, N., Amit, I., and Schuldiner, O. (2018). Combining Developmental and Perturbation-Seq Uncovers Transcriptional Modules Orchestrating Neuronal Remodeling. Dev Cell 47, 38-52 e36.

Diao, F., Ironfield, H., Luan, H., Diao, F., Shropshire, W.C., Ewer, J., Marr, E., Potter, C.J., Landgraf, M., and White, B.H. (2015). Plug-and-play genetic access to drosophila cell types using exchangeable exon cassettes. Cell Rep 10, 1410-1421.

Gratz, S.J., Ukken, F.P., Rubinstein, C.D., Thiede, G., Donohue, L.K., Cummings, A.M., and O'Connor-Giles, K.M. (2014). Highly specific and efficient CRISPR/Cas9-catalyzed homology-directed repair in Drosophila. Genetics 196, 961-971.

Heinz, S., Benner, C., Spann, N., Bertolino, E., Lin, Y.C., Laslo, P., Cheng, J.X., Murre, C., Singh, H., and Glass, C.K. (2010). Simple combinations of lineage-determining transcription factors prime cis-regulatory elements required for macrophage and $\mathrm{B}$ cell identities. Mol Cell 38, 576-589.

Kim, D., Langmead, B., and Salzberg, S.L. (2015). HISAT: a fast spliced aligner with low memory requirements. Nat Methods 12, 357-360.

Lee, T., and Luo, L. (1999). Mosaic analysis with a repressible cell marker for studies of gene function in neuronal morphogenesis. Neuron 22, 451-461.

Love, M.I., Huber, W., and Anders, S. (2014). Moderated estimation of fold change and dispersion for RNA-seq data with DESeq2. Genome Biol 15, 550.

Port, F., Chen, H.M., Lee, T., and Bullock, S.L. (2014). Optimized CRISPR/Cas tools for efficient germline and somatic genome engineering in Drosophila. Proc Natl Acad Sci U S A 111, E2967-2976.

Rabinovich, D., Yaniv, S.P., Alyagor, I., and Schuldiner, O. (2016). Nitric Oxide as a Switching Mechanism between Axon Degeneration and Regrowth during Developmental Remodeling. Cell 164, 170-182.

Vert, J.P., Foveau, N., Lajaunie, C., and Vandenbrouck, Y. (2006). An accurate and interpretable model for siRNA efficacy prediction. BMC Bioinformatics 7, 520 . 